\title{
THE EFFECT OF A SMALL INITIAL DISTORTION OF THE BASIC FLOW \\ ON THE SUBCRITICAL TRANSITION IN PLANE POISEUILLE FLOW
}

BY

PU SUN

Ford Motor Company, Ford Research Laboratory, Dearborn, MI

\begin{abstract}
This paper presents an instability theory in which a mean flow and multiple wave interactions in the Poiseuille flow transition process are studied. It is shown that not only can this mean flow term come as the result of the Fourier decomposition of a general disturbance, it can also come as an exact solution to the unsteady Navier-Stokes equations. The presence of this term, though small, can produce totally different linear and nonlinear stability behavior for the flow at subcritical Reynolds numbers. In the linear stability case, with the presence of this mean flow perturbation term, the instabilities are obtained well below the critical value of 5772 . When this mean flow term is introduced into the interactions with other harmonic perturbation waves, for the plane Poiseuille flow case with Reynolds numbers around 1200, the nonlinear interactions rapidly modify the total mean flow profile toward the mean flow profile observed in turbulence while the other two- and three-dimensional waves remain small. The initial energies needed to trigger the instabilities are much smaller than those reported by previous investigators. The intermittent character of the disturbance observed in transition experiments is also captured.
\end{abstract}

1. Introduction. The problem of the transition from laminar to turbulent flow has attracted much attention and has become one of the fundamental theoretical problems in fluid dynamics. Though considerable theoretical and experimental developments have been achieved, gaps still exist between the experimental observations and the theoretical and numerical study results. In the pipe flow experiments, if finite amplitude perturbations are introduced or tubes with roughened walls are used, the critical Reynolds number $R_{c}$ can be as low as 2000. As for the plane Poiseuille flow, transition of the laminar flow to turbulence is observed to occur explosively at Reynolds numbers as low as roughly 1000 . On the other hand, in the experiment by Nishioka et al [1], it was 
found that plane Poiseuille flow could remain laminar at Reynolds numbers up to 8000 by reducing the background turbulence at the intake.

The linear stability theory: which yields the Orr-Sommerfeld (OS) equation. studies the development of infinitesimal perturbation waves of the form

$$
(u, v, w)=(\hat{u}(y), \hat{v}(y), \hat{w}(y)) e^{i(\alpha x+\beta z-\alpha c t)} .
$$

However, for the plane Poiseuille flow case, the critical Reynolds number of the OS equation has the value 5772, which is much higher than the critical Reynolds number observed in experiments.

On the nonlinear stability aspect, a nonlinear equation

$$
\frac{d|A|^{2}}{d t}=k_{1}|A|^{2}+k_{2}|A|^{4}
$$

was originally conjectured by Landan [2] to describe the evolution of the amplitude of perturbation waves, but he offered no analysis. The systematic investigation of the nonlinear stability behavior of the shear flows was first carried out by Mcksyn and Stuart [3], and Stuart [4]. Stuart is the first to derive Eq. (1.1) together with the coefficients $k_{1}$ and $k_{2}$ by amplitude expansion techniques to study the nonlinear hydrodynamic instabilities. The nonlinear stability theory of two-dimensional laminar flow stems from this work and later from the work of Stuart [5] and Watson [6]. These results suggest that the finite amplitude of the perturbation waves may be the source of subcritical instability of the shear flows. In the plane Poiseuille flow case, subcritical instability means that the instabilities happened below the linear critical Reynolds number 5772. Reynolds and Potter [7] applied the Stuart and Watson theory to plane Poiseuille flow and showed that nonlinear effects have a destabilizing influence on the flow, and the same conclusion was also arrived at by Pekeris and Shkoller [8]. Generally speaking, the aim of these approaches is to derive a (1.1)-type equation for the perturbation amplitude. In the application of this approach, the derivation can be successfully carried out for the supercritical region where $R \geq 5772$, but without modifications there are difficulties in the subcritical region $R \leq 5772$. Another set of papers, namely those of Meksyn and Stuart [3], Grohne [9], and others, used the so-called mean-field equations to find the relationship between the critical Reynolds number $\left(R_{c}\right)$ and the threshold amplitude of the disturbance waves. It was shown that typically a root-mean square velocity of about 8 percent corresponds to a value of $R_{c}$ of about 25003000 .

Direct numerical simulations of the nonlinear stability problem have developed rapidly in recent decades. By representing the solution with a few Fourier modes in the streamwise direction and a finite difference mesh in the cross-stream direction, Zang and Krist [10] directly solved the Navier-Stokes equations. It was found that various initial conditions led either to complete decay of the disturbance or to a finite amplitude equilibrated wave. Higher-order approximation computations were performed by Herbert [11, 12] employing spectral methods, and the same results were obtained. These results were confirmed by Wolf et al [13]. Orszag and Patera [14], Kleiser and Schumann [15] and others. On the other hand. Orszag and Kells [16] computed the transition to turbulence in plane Poiseuille and plane Couette flow directly from the two- and three-dimensional disturbances. By using two-dimensional waves initially with amplitude above 0.08 (the 
maximum of the basic flow velocity is assumed to be one) and three-dimensional waves initially with amplitude larger than 0.04, subcritical instability was found. Based on these computations, Orszag and Patera [17] and Herbert [18] put forth the secondary instability theory. They investigated the stability of three-dimensional waves on a basic Poiseuille flow plus a two-dimensional equilibrated wave. Rapidly growing instabilities were obtained, and the critical Reynolds number, computed for the instability of the infinitesimal three-dimensional wave, corresponded very well to the Reynolds number at which transition occurred. A more detailed discussion of the secondary instability theory can be found in the review article by Bayly, Orszag and Herbert [19].

Benney and Lin [20] considered the mean flow induced by the interaction of a twoand a three-dimensional disturbance. Their results showed that many of the essential qualitative features of the experiments of Klebanoff et al [21] can be reproduced. Also, Benney [22] proposed a theory for the evolution of three-dimensional waves on a shear flow in which it was shown that the three-dimensionality of perturbation waves plays a crucial role in the generation and evolution of the mean flow terms.

In this paper, the effect of a small initial distortion of the mean flow on the linear and nonlinear stabilities of the shear flow is investigated. Throughout we investigate these effects on the plane Poiseuille flow case, for which a general and extensive discussion on the stability problem can be found in DiPrima and Stuart [23]. In this paper, we will show that not only can this mean flow distortion term come as the result of the Fourier decomposition of a general disturbance, it can also come as an exact solution to the unsteady Navier-Stokes equations. The presence of this term, though small, can produce totally different linear and nonlinear stability behavior for the flow at subcritical Reynolds numbers. Specifically, the linear instability is obtained at Reynolds numbers well below 1000. In the nonlinear case, the interactions between this mean perturbation term and the other two- and three-dimensional waves rapidly modify the total mean flow profile toward the mean flow profile observed in turbulence. In Sec. 2, the mean flow modification is introduced. The linear stability is investigated and the results are shown in Sec. 3. In Sec. 4, the nonlinear effects are analyzed, and then a set of weakly nonlinear equations governing the development of the various components of the perturbations is derived in Sec. 5. The computational results of the governing equations derived in Sec. 5 are presented and discussed in Sec. 6. We draw the conclusion in Sec. 7.

2. Mean flow modifications. In $x, y$ coordinates, the two-dimensional nondimensionalized Navier-Stokes equations and the continuity equation are as follows:

$$
\begin{aligned}
& \frac{\partial \mathbf{v}}{\partial t}+\mathbf{v} \cdot \nabla \mathbf{v}=-\nabla p+\frac{1}{R} \Delta \mathbf{v}, \\
& \nabla \cdot \mathbf{v}=0
\end{aligned}
$$

where velocity $\mathbf{v}=(u, v), p$ is pressure, and $x, y$, and $t$ are all nondimensionalized with respect to the reference velocity, $U_{c}$, reference pressure, $\rho U_{c}^{2}$, reference length, $l$, and reference time, $l / U_{c} . \quad R=U_{c} l / \nu$ denotes the Reynolds number based upon these reference quantities. For our plane Poiseuille flow case, $l$ is the half-width of the channel, $U_{c}$ is the centerline velocity, $\rho$ is the density of the fluid, and $\nu$ is the kinematic viscosity 


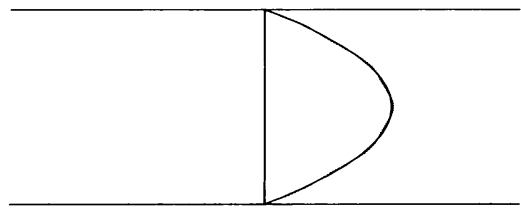

FIG. 1. Profiles of Poiseuille flow $U=1-y^{2}$ (solid line) and the unsteady Poiseuille basic flow $\bar{U}=1-y^{2}+a \cos \pi(k-1 / 2) y, a=$ $0.015, k=5$ (dotted line).

of the fluids. We take $x$ and $y$ as the streamwise and vertical (cross-stream) coordinates so that the boundary conditions are $\mathbf{v}(x, y= \pm 1)=0$. Suppose that

$$
v=\frac{\partial u}{\partial x}=\frac{\partial p}{\partial y}=0
$$

in which case the only equation left to solve is

$$
\frac{\partial u}{\partial t}=-\frac{\partial p}{\partial x}+\frac{1}{R} \frac{\partial^{2} u}{\partial y^{2}}
$$

This equation differs from the equations that give us the plane Poiseuille flow by its time-derivative term. It is natural to expect that any real flow, even at low Reynolds numbers, may not be so pure that only the steady part exists. The solutions to Eq. (2.2) are

$$
\begin{aligned}
\bar{U} & =1-y^{2}+\bar{u}_{0}, \\
\bar{u}_{0} & =\sum a_{k} e^{-\left(k \pi-\frac{\pi}{2}\right)^{2} t / R} \cos \left[\left(k \pi-\frac{\pi}{2}\right) y\right]+\sum b_{k} e^{-(k \pi)^{2} t / R} \sin [k \pi y] .
\end{aligned}
$$

Notice that $\bar{U}$ in (2.3) is completely arbitrary since the $a_{k}$ 's and $b_{k}$ 's in $\bar{u}_{0}$ are arbitrary. But if $\left|\bar{u}_{0}\right|$ is small compared with $1-y^{2},(2.3)$ can be thought of as a basic state of a flow very close to the steady Poiseuille flow, which we refer to as an unsteady Poiseuille basic flow.

Figure 1 shows the comparison of velocity profiles between $U=1-y^{2}$ and the unsteady Poiseuille basic flow $\bar{U}$ with $\bar{u}_{0}(t=0, y)=a \cos \pi(k-1 / 2) y$, where $a=0.015$ and $k=5$. The solid line is for $U$ and the dotted line is for $\bar{U}$. It can be seen that the profile for $\bar{U}$ is still like a parabola. In the experiments, data are collected at a finite number of points, and the difference between a pure parabola and a composite velocity is by no means obvious.

The fact that Eqs. (2.3) are exact solutions of the Navier-Stokes equations indicates the possibility that the velocity profile tested in a two-dimensional transition experiment is actually $\bar{U}$ in (2.3) that includes a nonzero small $\bar{u}_{0}$. If so, the question we should answer is what effect will this mean flow perturbation term have on the stability characteristics of the flow.

Therefore, taking $\bar{U}$ with a nonzero but small $\bar{u}_{0}$ as the basic flow, we consider the following linear stability problem with the whole velocity

$$
\mathbf{V}=(\bar{U}+u, v)=\mathbf{i} \bar{U}+\mathbf{v} .
$$


with $\mathbf{i}$ as the unit vector in the $x$ direction and with $\mathbf{v}$ a two-dimensional disturbance so that $\mathbf{v} / \bar{U} \sim \varepsilon$, where $\varepsilon$ is a small quantity. Substituting in the Navier-Stokes equation, subtracting the basic equation of $\bar{U}$, namely (2.2), and neglecting all the nonlinear terms yields

$$
\begin{aligned}
& \frac{\partial \mathbf{v}}{\partial t}+\bar{U} \frac{\partial \mathbf{v}}{\partial x}+\mathbf{i} v \bar{U}^{\prime}=-\nabla p+\frac{1}{R} \Delta \mathbf{v}, \\
& \nabla \cdot \mathbf{v}=0, \\
& u=v=0, \quad y= \pm 1 .
\end{aligned}
$$

The only difference between (2.4) and the Orr-Sommerfeld equation is that the basic flow $\bar{U}$ in (2.4) is a function of both $t$ and $y$. Also, in order to determine $\bar{U}$, the constants in the expression of $\bar{u}_{0}$ need to be fixed. There are a great variety of choices for the $a_{k}$ 's and $b_{k}$ 's. In this paper, no complete analysis is attempted, and only the first few $a_{k}$ 's and $b_{k}$ 's are considered to demonstrate the possibilities. Therefore, in the following, we further simplify the expression of $\bar{u}_{0}$ by including only one term from each of its sine and cosine series. Thus, $\bar{u}_{0}$ becomes

$$
\bar{u}_{0}=a e^{-\left(k \pi-\frac{\pi}{2}\right)^{2} t / R} \cos \left[\left(k \pi-\frac{\pi}{2}\right) y\right]+b e^{-(k \pi)^{2} t / R} \sin [k \pi y] .
$$

3. The effect on the linear stability characteristics. In order to determine what effect the mean flow perturbation term introduced in Sec. 2 will have on the linear stability characteristics of the flow, the solution to the linearized Navier-Stokes equation is investigated in this section. Since the basic flow $\bar{U}$ in (2.4) is a function of both $t$ and $y$, a normal mode approach as used in the OS equation is no longer applicable. However, if we consider the situation in which $R$ is large and only the first few sine and cosine modes are included in $\bar{u}_{0}$, the decay of the amplitudes is slow (see (2.5)). Therefore, we may first assume that $\bar{u}_{0}$ has constant $a$ and $b$ as amplitudes of its sine and cosine modes, and then we may use the same normal mode approach as in the OS equation. In this way, the computational method used to solve the OS equation can be employed to solve (2.4), and the eigenvalues in each case can readily be compared. For the time-dependent case, that is $\bar{u}_{0}=\bar{u}_{0}(t, y)$, we will explicitly compute the time-dependent solutions of (2.4) and study their evolutions.

3.1. Eigenvalue computations. Under the assumption that $\bar{u}_{0}$ has constant amplitudes in its sine and cosine modes in (2.5), we have

$$
\bar{U}=U+\bar{u}_{0}=U+a \cos \left[\left(k \pi-\frac{\pi}{2}\right) y\right]+b \sin [k \pi y]
$$

and we seek a solution of the form

$$
(u, v, p)=(\hat{u}(y), \hat{v}(y), \hat{p}(y)) e^{i \alpha(x-c t)},
$$




\begin{tabular}{|lllll|}
\hline $\bar{U}=1-y^{2}$ & $R=2000$ & $\alpha=1$ & $a=0$ & $b=0$ \\
\hline & Chebyshev & tau & Chebyshev & collocation \\
\hline & $\alpha c_{r}$ & $\alpha c_{i}$ & $\alpha c_{r}$ & $\alpha c_{i}$ \\
\hline$M=15$ & 0.312100 & -0.0197990 & 0.312100 & -0.0197993 \\
$M=20$ & 0.312100 & -0.0197986 & 0.312100 & -0.0197986 \\
$M=25$ & 0.312100 & -0.0197986 & 0.312100 & -0.0197986 \\
\hline
\end{tabular}

TABLE 1

\begin{tabular}{|lllll|}
\hline $\bar{U}=1-y^{2}+\bar{u}_{0}$ & $R=2000$ & $\alpha=1$ & $a=-0.03$ & $k=3$ \\
\hline & Chebyshev & tau & Chebyshev & collocation \\
\hline & $\alpha c_{r}$ & $\alpha c_{i}$ & $\alpha c_{r}$ & $\alpha c_{i}$ \\
\hline$M=12$ & 0.306025 & 0.0157807 & 0.306031 & 0.0157813 \\
$M=15$ & 0.306035 & 0.0157795 & 0.306035 & 0.0157754 \\
$M=20$ & 0.306035 & 0.0157754 & 0.306035 & 0.0157754 \\
$M=25$ & 0.306035 & 0.0157754 & 0.306035 & 0.0157754 \\
\hline
\end{tabular}

TABLF: 2

so that (2.4) becomes

$$
\begin{aligned}
i \alpha(\bar{U}-c) \hat{u}+\hat{v} \bar{U}^{\prime} & =-i \alpha \hat{p}+\frac{1}{R}\left(\frac{\partial^{2}}{\partial y^{2}}-\alpha^{2}\right) \hat{u}, \\
i \alpha(\bar{U}-c) \hat{v} & =-\frac{\partial \hat{p}}{\partial y}+\frac{1}{R}\left(\frac{\partial^{2}}{\partial y^{2}}-\alpha^{2}\right) \hat{v} \\
i \alpha \hat{u}+\frac{\partial \hat{v}}{\partial y} & =0, \\
\hat{u}=\hat{v} & =0, \quad y= \pm 1 .
\end{aligned}
$$

Both the Chebyshev tau and Chebyshev collocation methods are used to solve the problem. In these methods, the unknown functions $\hat{u}, \hat{v}$ and $\hat{p}$ are expanded in terms of Chebyshev polynomials $T_{m}, m=0,1,2, \ldots, M$,

$$
\hat{u}=\sum_{m=0}^{M} \hat{u}_{m} T_{m}, \quad \hat{v}=\sum_{m=0}^{M I} \hat{v}_{m} T_{m} . \quad \hat{p}=\sum_{m=0}^{M-1} \hat{p}_{m} T_{m} .
$$

In order to test the convergence and accuracy, first the program is run with $\bar{u}_{0}=0$, in which case the problem is equivalent to the original OS equation. Table 1 shows the convergence bchavior and accuracy. These results are in good agreement with published data. Next, we include small mean flow perturbations in $\bar{U}$ and run the program again. Surprisingly, at Reynolds numbers as low as 2000, instability was found and the growth rates $\alpha c_{i}$ are not very small. The results are illustrated in Table 2.

Also, it is casy to see that the results from the Chebyshev tau and the Chebyshev collocation method agree very well, and this leads us to believe that these results are reliable. For other situations, Figures 2 and 3 show the growth rates $\alpha c_{i}$ and $\alpha c_{r}$ values 

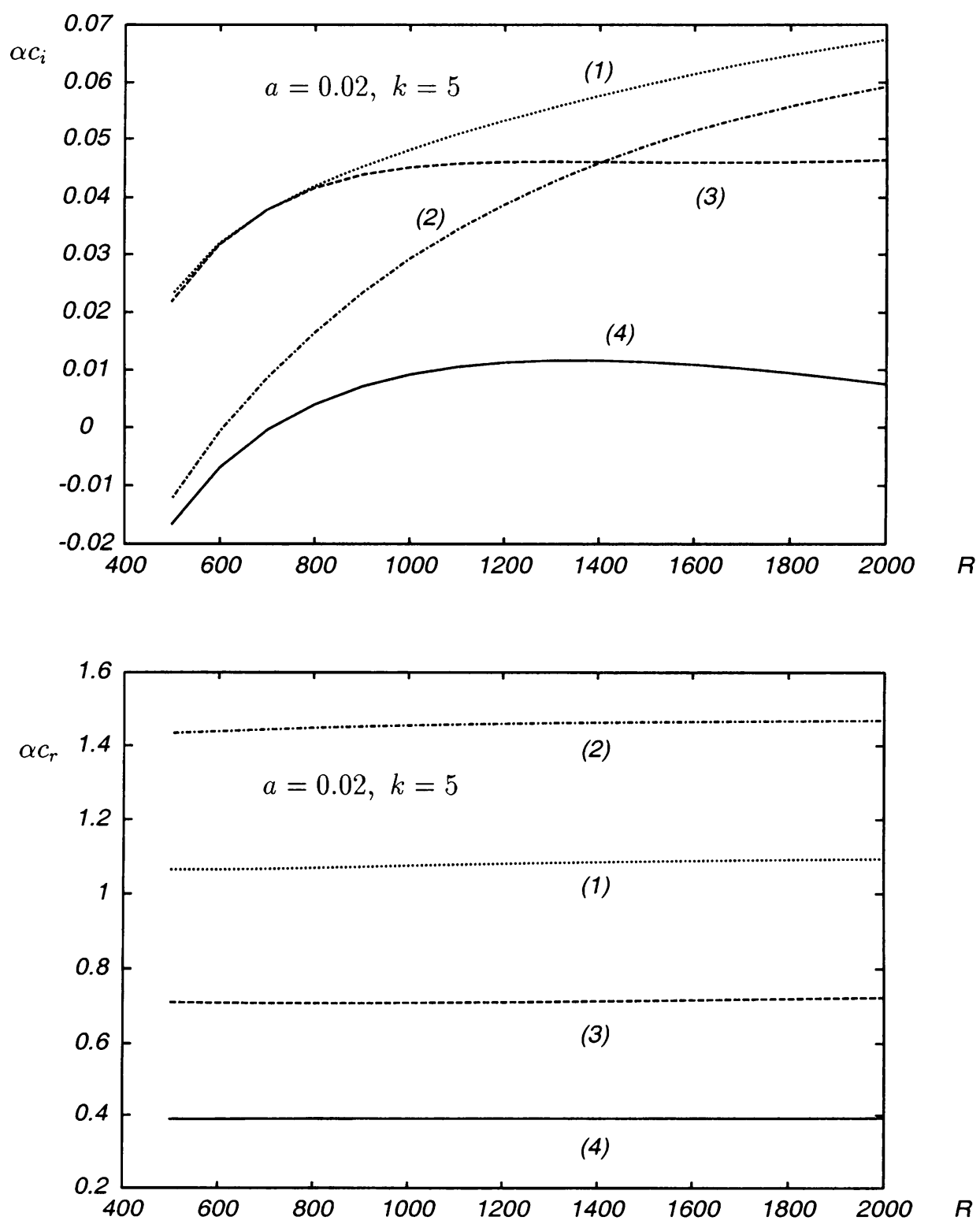

FIG. 2. Growth rate $\alpha c_{i}$ and $\alpha c_{r}$ values for $\bar{U}=1-y^{2}+a \cos \pi(k-$ 1/2)y. (1): $\alpha=2$, (2) $\alpha=2.5,(3): \alpha=1.5,(4): \alpha=1$.

at Reynolds numbers from 500 to 2000 computed for unsteady plane Poiseuille basic flow by the Chebyshev tau method. The expansion number $M$, as in (3.4), used is 30 . It is seen that the instabilities are found at Reynolds numbers as low as 500. The amplitudes $a$ and $b$ can be as low as 0.02 . 


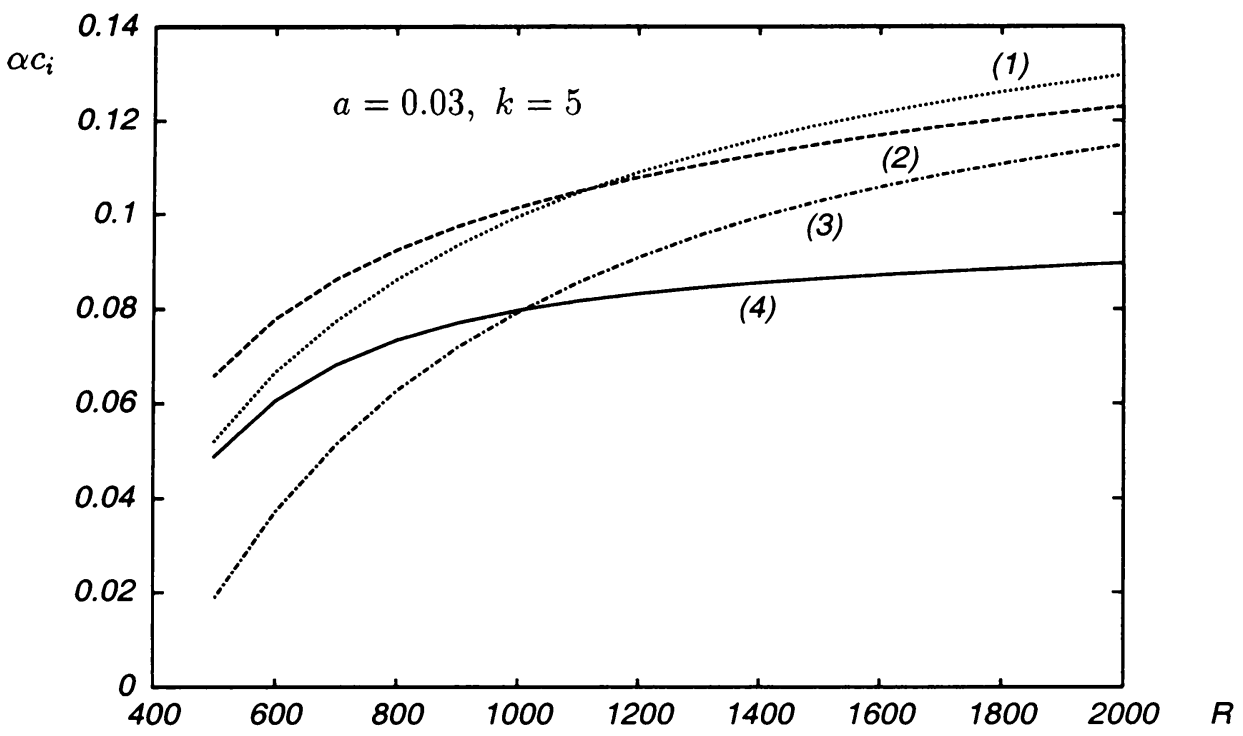

FIG. 3. Growth rate $\alpha c_{i}$ and $\alpha c_{r}$ values for $\bar{U}=1-y^{2}+a \cos \pi(k-$ 1/2)y. (1): $\alpha=2.5,(2): \alpha=2,(3): \alpha=3,(4): \alpha=1.5$.

The results of this section are believed to be important since they shed more light on the understanding of the subcritical instabilities. They show that the puzzling subcritical instability is actually not difficult to obtain if a small mean flow perturbation term is included into the basic steady mean flow. Also, they suggest a possible explanation for the transition experiment. That is, it is the presence of a small $\bar{u}_{0}$ in the mean flow profile that makes the flow become unstable at a Reynolds number well below 5770 .

3.2. The time-dependent case. The instability in the constant-amplitude case is an estimate. When the amplitude of $\bar{u}_{0}$ varies with time, we may expect that instabilities may occur so long as $a$ and $b$ are above the values used in the computation in Sec. 3.1 for the corresponding Reynolds numbers. However, as the amplitudes $a$ and $b$ decay with time, the flow should cease to be unstable. The time-dependent amplitudes case is investigated in this subsection.

For simplicity we rewrite the Navier-Stokes equation in (2.4) as

$$
\frac{\partial \mathbf{v}}{\partial t}=G(\bar{U}, \mathbf{v}, p)
$$

Then we expand the unknown functions in the form

$$
f(t, x, y)=e^{i \alpha x} \sum_{m=0}^{M} f_{m}(t) T_{m}(y), \quad f=u, v, p,
$$




\begin{tabular}{|llllll|}
\hline $\bar{U}=1-y^{2}$ & $R=2000$ & $\alpha=1$ & $\Delta t=0.1$ & $\bar{u}_{0}=0$ & \\
\hline$t$ & 2 & 4 & 6 & 8 & 10 \\
\hline $\log [E n(t)]$ & -0.0791 & -0.1583 & -0.2375 & -0.3167 & -0.3958 \\
$2 \alpha c_{i} t$ & -0.0791 & -0.1583 & -0.2375 & -0.3167 & -0.3959 \\
\hline $\bar{U}=1-y^{2}+\bar{u}_{0}$ & $a=-0.03$ & $k=3$ & $b=0$ & $\Delta t=0.1$ & \\
\hline$t$ & 2 & 4 & 6 & 8 & 10 \\
\hline $\log [E n(t)]$ & 0.0630 & 0.1261 & 0.1892 & 0.2523 & 0.3154 \\
$2 \alpha c_{i} t$ & 0.0631 & 0.1262 & 0.1893 & 0.2524 & 0.3155 \\
\hline
\end{tabular}

TABLE 3

and use the Crank-Nicolson scheme for the time advancement. After these procedures, (3.5) and the continuity equation reduce to

$$
\begin{aligned}
\frac{\mathbf{v}^{n+1}-\mathbf{v}^{n}}{\Delta t} & =\left(G^{n+1}+G^{n}\right) / 2, \\
\nabla \cdot \mathbf{v}^{n+1} & =0 .
\end{aligned}
$$

With the homogeneous boundary condition $u(t, \pm 1)=v(t, \pm 1)=0$ and the initial values of $u, v$, and $p$ being the most unstable modes from the computation of the last subsection, Eq. (3.6) is then solved by the Chebyshev tau method. Two runs are made to verify the accuracy in comparison with that predicted by the eigenvalue computation. In the first run, $\bar{u}_{0}=0$ and the least stable eigenmode from the solution to the OS equation is chosen to be the initial value. In the second run, the coefficients $a$ and $b$ in $\bar{u}_{0}$ are assumed to be constants. In this case, we expect that when the truncation error from the finite difference in time in (3.6) is small, the logarithm of the relative energy of the oblique wave $(u, v)$, which is defined below,

$$
E n(t)=e n(t) / e n(0), \quad e n(t)=\int_{-1}^{1}\left(|u|^{2}+|v|^{2}\right) d y
$$

should be very close to the straight line $2 \alpha c_{i} t$, where $\alpha c_{i}$ is computed from (3.4) by the eigenvalue computation method in the last subsection for the same least stable eigenmode. Table 3 shows the results from these two comparison runs with the expansion number $M=20$. The upper part of Table 3 shows $\log [E n(t)]$ from the computation of (3.6) in comparison with $2 \alpha c_{i} t$ from the eigenvalue computation in Sec. 3.1 at $\bar{u}_{0}=0$. The same comparisons, with $\bar{u}_{0}=a \cos [(k \pi-\pi / 2) y]$, are shown in the lower part of Table 3 . Also, the results from the Chebyshev tau method are found to be almost identical with those from the Chebyshev collocation method, and it is believed that the time difference error is small and that these codes are correct. After these procedures, the energy evolvement for the time-dependent $\bar{u}_{0}$ case is carried out by a Chebyshev tau method and Fig. 4 shows the results. In the figure, the graph of the logarithms of the energies $E n(t)$, as in (3.7), for the time-dependent $\bar{u}_{0}$ case is labeled (T), and that from steady $\bar{u}_{0}$ (constant $a$ 's and $b$ 's) is labeled (S). It is interesting to see how much a normal mode can be amplified before it begins to decay. In Fig. 4, with $a=0.03$ at $t=0$ and $k=5$, the energy of an initial normal mode can grow to about $e^{1.6} \doteq 5$ times its original value. 


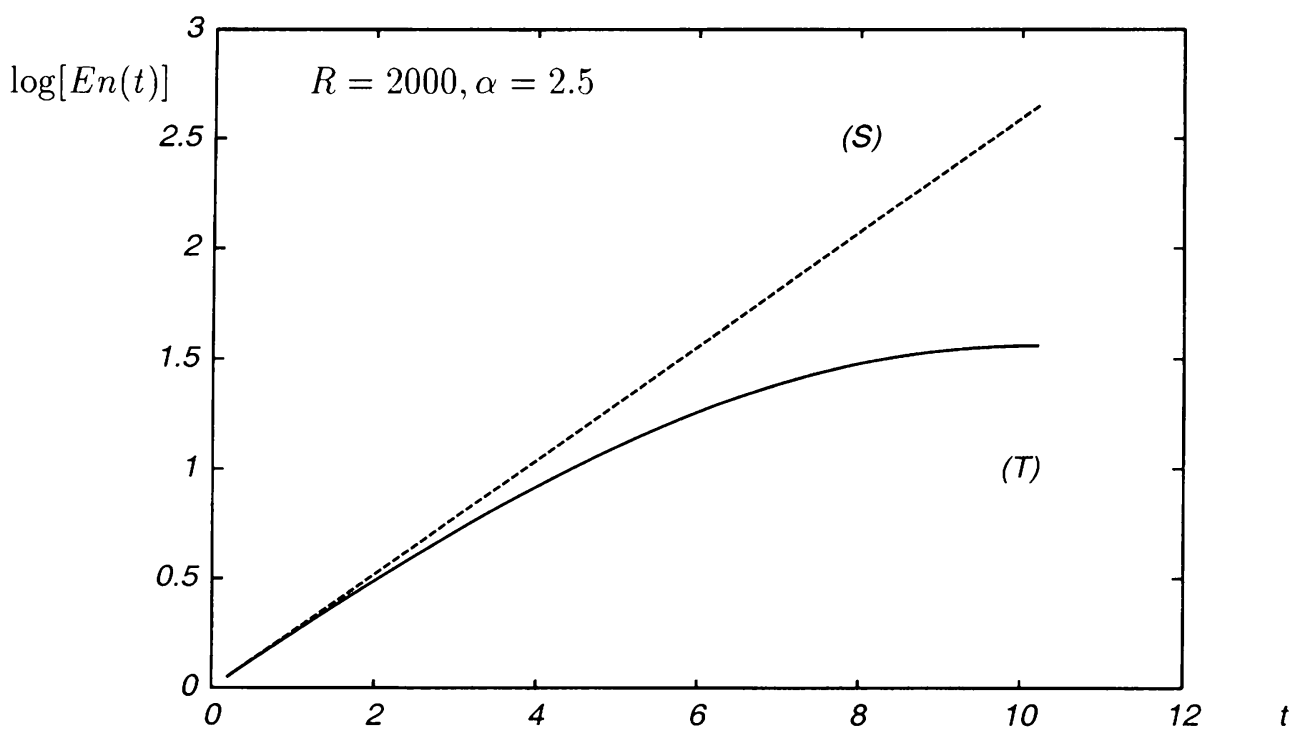

FIC. 4. Comparison of the energy evolvement for $\bar{U}$ with unsteady and steady $\bar{u}_{0}$. (T): $\bar{L}=\bar{U}(t . y),(\mathrm{S}): \bar{U}=\bar{U}(y), a=0.03, k=5$.

The linear instabilities obtained in this section show that the original Orr-Sommerfeld equation is sensitive to small alterations to the mean flow profile. The fact that this mean flow perturbation term is an exact solution to the Navier-Stokes equations indicates that this term can directly come to the flow field as the result of some unsteady background disturbances in the flow. Though the low Reynolds number instabilities can be obtained only when the amplitude of $\bar{u}_{0}$ is above a small value, many other changes can take place before $\bar{u}_{0}$ decays below this small value when a nonlinear or weakly nonlinear theory is considered, when initially $\bar{u}_{0}$ is above this small value. The shift of the critical Reynolds number from 5770 to a value well below 1000 leaves much room for a nonlinear or a wakkly nonlinear theory to come into play.

4. Analysis on the nonlinear stability characteristics. In many previous numerical investigations, the full Navier-Stokes equations were computed. In these computations, the initial conditions of the perturbation velocities mainly belong to two categories. In the first category, all the perturbation velocities are taken from the least stable or the most unstable two-dimensional or three-dimensional eigenfunctions of the OS equations, such as those of Orszag and Kells [16] and Zang and Krist [10]. In the second category, the initial perturbation velocities consist of a two-dimensional equilibrated wave and the least stable three-dimensional eigenfunctions of the OS equation, such as those of Orszag and Patera [14] and Herbert [18] in the so-called secondary instability theory. While instabilities were found and the transition processes were observed, the amplitudes of these initial perturbation velocities needed to trigger instabilities at Reynolds numbers of 1000-2000 were by no means small. In Orszag and Kells [16], the 
initial $x$-direction two-dimensional velocity has an amplitude of $0.08-0.11$, and the threedimensional $x$-direction velocity has an amplitude of $0.04-0.05$. Also, in the secondary instability computations by Orszag and Patera [14], initial amplitudes corresponding to a root mean square maximum perturbation streamwise velocity of about $20 \%$ of the basic flow centerline velocity were used. If fairly small initial perturbations were used, no instability or transition would be obtained. On the other hand, at the same Reynolds number range, the instability and transition were observed in experiments where the initial perturbations introduced into the flow were much smaller.

In Sec. 3, it was shown that when a small unsteady term $\bar{u}_{0}$ is included in the basic plane Poiseuille flow, instability often arises and the critical Reynolds number can be as low as 500. The initial conditions based upon the inclusion of $\bar{u}_{0}$ differ from the two categories of the initial conditions previously used. The sharp drop of the critical Reynolds number in the linear theory investigation of the plane Poiseuille flow makes us suspect that the inclusion of this term in a nonlinear theory may also make the flow more unstable. We also suspect that the decay of this term in the linear theory, where all other perturbation waves are infinitesimal, may no longer be the case in a nonlinear theory. In this section, we analyze these possibilitics and demonstrate the existence of the possible mechanism.

A general form for the unidirectional unsteady part is

$$
\bar{u}_{0}=\sum a_{k} e^{-\left(k \pi-\frac{\pi}{2}\right)^{2} t / R} \cos \left[\left(k \pi-\frac{\pi}{2}\right) y\right]+\sum b_{k} e^{-(k \pi)^{2} t / R} \sin [k \pi y] .
$$

The amplitudes $a_{k} e^{-\left(k \pi-\frac{\pi}{2}\right)^{2} t / R}$ and $b_{k} e^{-(k \pi)^{2} t / R}$ are arbitrary but are considered to be initially small so that the composite total velocity is a perturbed form of the steady part. We can assume $\bar{u}_{0}=O(\varepsilon)$.

We use the model equation below to investigate the role $\bar{u}_{0}$ plays in a nonlinear theory:

$$
\frac{\partial u}{\partial t}=i \sigma \frac{\partial u}{\partial x}+u^{2}, \quad \sigma \geq 0 .
$$

Though simple, this model equation essentially has the same structure as the equation governing the development of a two-dimensional disturbance stream function and thus the study of the equation can reveal many physical characteristics of a real flow.

Consider an expansion

$$
u=\sum y_{n} e^{i \alpha n x}+\sum y_{n}^{*} e^{-i \alpha n x}, \quad y_{n} \sim|A|^{n}, \quad n \geq 1 .
$$

When $u$ is substituted into the equation and the Fourier modes are equated, the ordinary differential equation for the amplitude $|A|$ can be found to have the form

$$
\frac{d|A|}{d t}=-\sigma \alpha|A|+|A|^{3}+O\left(|A|^{5}\right)
$$

If $|A|$ is small, the higher-order terms can be neglected and the above equation can be written as

$$
\frac{d|A|^{2}}{d t}=-2 \sigma \alpha|A|^{2}+2|A|^{4} .
$$

This is the Landau equation. In its linearized form, $|A|^{2}$ decays. But if $\sigma$ is small, $|A|^{2}$ could grow in the nonlinear case depending on whether $-2 \sigma \alpha|A|^{2}+2|A|^{4}$ is positive 
or negative. It is obvious that when $|A|^{2} \sim \sigma \alpha,-2 \sigma \alpha|A|^{2}+2|A|^{4}$ could be positive. However, in hydrodynamic stability problems, $\sigma \alpha$ is the linear decay rate $\alpha c_{i}$. If the perturbation amplitude $A$ is order of $\varepsilon$, the possibility for the amplitude $|A|$ to grow requires that $\alpha c_{i} \sim \varepsilon^{2}$. Therefore. $\alpha c_{i}$ should be very small, and thus the application of the Landau equation is restricted to a narrow vicinity of the so-called critical Rernolds number $R_{c}=5770$, where $\alpha c_{i} \sim \Xi^{2}$. This strict limitation makes the Landau equation incapable of describing the nonlinear instability problems at Reynolds numbers much below $R_{c}$.

But, if instead of the expression $u=\sum y_{n} e^{i \alpha n x}+\sum y_{n}^{*} e^{-i \alpha n x}$. we have

$$
u=\bar{u}_{0}+\sum y_{n} e^{i \alpha n x}+\sum y_{n}^{*} e^{-i \alpha n x}, \quad y_{n} \sim|A|^{n}, \quad n \geq 1,
$$

where $\bar{u}_{0} \sim \varepsilon$, the amplitude equation will look like this:

$$
\frac{d|A|}{d t}=-\sigma \alpha|A|+\bar{u}_{0}|A|+O\left(|A|^{3}\right) .
$$

In this equation, the condition for the possible growth of $|A|$ is $\sigma a \sim \bar{u}_{0}$. Namely the condition for possible growth of the wave has been changed from $\sigma \alpha \sim \varepsilon^{2}$ in Landau's equation case to $\sigma \alpha \sim \Xi$ here. This important change has opened a way to use this mechanism to study nonlinear stability problems at Reynolds numbers much lower than $R_{c}$, where $\sigma \alpha \sim \varepsilon$ is often the case, as was shown in the linear stability characteristic studies. Generally speaking, for the plane Poiseuille flow. at $R \geq 1200$, the linear decay rate $\alpha c_{i}$ of the least stable mode can be considered to be of order $\varepsilon$. Therefore, in such situations, it will be possible for the two- and three-dimensional finite waves to become unstable in a nonlinear theory. Furthermore, the hydrodynamic stability equations are much more complicated than the model equation above, and it is quite possible that the inclusion of $\bar{u}_{0}$ will have a much larger nonlinear effect in the hydrodymamic stability equations than in the above model equation.

The next question we need to answer is. "can $\bar{u}_{0}$ persist?" We know that in a long twodimensional channel, the laminar flow velocity profile is a parabola. whereas for turbulent flow, the mean flow profile is flatter in the vicinity of the centerline and changes more rapidly in the region close to the wall. During the transition process, the mean velocity profile changes from its laminar shape to its turbulent shape. In order for this substantial change to occur, instead of decaying, $\bar{u}_{0}$ must be sustained and grow. Intuitively, we expect that nonlinearity may make this mechanism possible if these oblique waves do not decay. Roughly speaking, in the evolution equation of $\bar{u}_{0}$, the contributions from the oblique waves are the self-interaction terms, which have the order of magnitude $\varepsilon^{2}$. The viscous term in the equation is $\frac{1}{R} \frac{\partial^{2} \bar{u}_{0}}{\partial y^{2}}$. Under the condition that $\frac{1}{R} \sim \varepsilon$ and $\bar{u}_{0} \sim \varepsilon$, the possibility that $\bar{u}_{0}$ will grow while changing its spatial structure certainly exists.

The analysis above indicates the importance of the initial appearance of $\bar{u}_{0}$ and a possible role the nonlinear terms play to sustain it. Accordingly, the evolution equations for $\bar{u}_{0}$ and oblique waves should include these nonlinear terms. On the other hand, according to the experiments, although the mean flow profile undergoes a substantial change during the transition process, other mechanical qualities remain small and fluctuate in an irregular, random manner in space and time. Based upon this fact, our evolution equations for $\bar{u}_{0}$ and other oblique waves will include second-order nonlinear terms, whereas the 
higher-order terms are neglected. In the situation where the waves grow, these equations will be valid until the waves reach a point where third-order terms are comparable with the second-order terms. If they decay, there will be no question about the validity of neglecting the third-order terms. It may be expected that if the physical behavior of the transition is to be correctly described, on the whole, the perturbation oblique waves should remain small and hence truncation at third-order is self-consistent. Also, since three-dimensionality plays an important role in the transition process, three-dimensional together with two-dimensional perturbation waves will be considered.

5. Nonlinear interaction governing equations. In order to describe the interactions between the mean flow perturbation and the oblique waves, we chose the following expressions for the velocities and pressure:

$$
\begin{aligned}
u= & U(y)+\bar{u}_{0}(y, t)+\varepsilon u_{0}(y, t) e^{2 i \beta z}+\text { c.c. } \\
& +\varepsilon\left(u_{+}(y, t) e^{i \beta z}+u_{-}(y, t) e^{-i \beta z}\right) e^{i \alpha x}+\text { c.c. }+\cdots, \\
v= & \varepsilon \bar{v}_{0}(y, t)+\varepsilon v_{0}(y, t) e^{2 i \beta z}+\text { c.c. } \\
& +\varepsilon\left(v_{+}(y, t) e^{i \beta z}+v_{-}(y, t) e^{-i \beta z}\right) e^{i \alpha x}+\text { c.c. }+\cdots, \\
w= & \varepsilon \bar{w}_{0}(y, t)+\varepsilon w_{0}(y, t) e^{2 i \beta z}+\text { c.c. } \\
& +\varepsilon\left(w_{+}(y, t) e^{i \beta z}+w_{-}(y, t) e^{-i \beta z}\right) e^{i \alpha x}+\text { c.c. }+\cdots, \\
p= & -\frac{2}{R} x+\varepsilon \bar{p}_{0}(y, t)+\varepsilon p_{0}(y, t) e^{2 i \beta z}+\text { c.c. } \\
& +\varepsilon\left(p_{+}(y, t) e^{i \beta z}+p_{-}(y, t) e^{-i \beta z}\right) e^{i \alpha x}+\text { c.c. }+\cdots .
\end{aligned}
$$

In (5.1), $x, y$, and $z$ are the streamwise, vertical (cross-stream), and spanwise directions, respectively. Also in (5.1), a quantity following $\varepsilon$ means that quantity is assumed to be of order of $\varepsilon$ and c.c. represents the complex conjugate of its preceding term.

Substituting (5.1) into the Navier-Stokes equations and the continuity equation and neglecting the third-order terms in $\varepsilon$, and also absorbing $\varepsilon$ into the corresponding quantities yields the sets of equations below:

$$
\begin{aligned}
& \frac{\partial \overline{\mathbf{v}}_{0}}{\partial t}+\overline{\mathbf{v}}_{0} \cdot \nabla \overline{\mathbf{v}}_{0}+\mathbf{v}_{0} \cdot \nabla \mathbf{v}_{0}^{*}+\mathbf{v}_{0}^{*} \cdot \nabla \mathbf{v}_{0}+\mathbf{v}_{+} \cdot \nabla \mathbf{v}_{+}^{*}+\mathbf{v}_{+}^{*} \cdot \nabla \mathbf{v}_{+} \\
& +\mathbf{v}_{-} \cdot \nabla \mathbf{v}_{-}^{*}+\mathbf{v}_{-}^{*} \cdot \nabla \mathbf{v}_{-}=-\nabla \bar{p}_{0}+\frac{1}{R} \Delta \overline{\mathbf{v}}_{0}, \quad \nabla \cdot \overline{\mathbf{v}}_{0}=0 \\
& \frac{\partial \mathbf{v}_{0}}{\partial t}+\overline{\mathbf{v}}_{0} \cdot \nabla \mathbf{v}_{0}+\mathbf{v}_{0} \cdot \nabla \overline{\mathbf{v}}_{0}+\mathbf{v}_{+} \cdot \nabla \mathbf{v}_{-}^{*}+\mathbf{v}_{-}^{*} \cdot \nabla \mathbf{v}_{+}=-\nabla p_{0}+\frac{1}{R} \Delta \mathbf{v}_{0} \\
& \nabla \cdot \mathbf{v}_{0}=0, \\
& \frac{\partial \mathbf{v}_{+}}{\partial t}+\overline{\mathbf{v}}_{0} \cdot \nabla \mathbf{v}_{+}+\mathbf{v}_{+} \cdot \nabla \overline{\mathbf{v}}_{0}+\mathbf{v}_{-} \cdot \nabla \mathbf{v}_{0}+\mathbf{v}_{0} \cdot \nabla \mathbf{v}_{-}=-\nabla p_{+}+\frac{1}{R} \Delta \mathbf{v}_{+}, \quad \nabla \cdot \mathbf{v}_{+}=0
\end{aligned}
$$




$$
\begin{aligned}
\frac{\partial \mathbf{v}_{-}}{\partial t}+\overline{\mathbf{v}}_{0} \cdot \nabla \mathbf{v}_{-}+\mathbf{v}_{-} \cdot \nabla \overline{\mathbf{v}}_{0}+\mathbf{v}_{+} \cdot \nabla \mathbf{v}_{0}^{*}+\mathbf{v}_{0}^{*} \cdot \nabla \mathbf{v}_{+}=-\nabla p_{-}+\frac{1}{R} \Delta \mathbf{v}_{-}, & \nabla \cdot \mathbf{v}_{-}=0,
\end{aligned}
$$

where

$$
\begin{aligned}
\overline{\mathbf{v}}_{0} & =\left(U+\bar{u}_{0}, \bar{v}_{0}, \bar{w}_{0}\right), & \mathbf{v}_{0} & =\left(u_{0}, v_{0}, w_{0}\right) e^{2 i \beta z}, \\
\mathbf{v}_{+} & =\left(u_{+}, v_{+}, w_{+}\right) e^{i \alpha x+i \beta z} . & \mathbf{v}_{-} & =\left(u_{-}, v_{-}, w_{-}\right) e^{i \alpha x-i \beta z} .
\end{aligned}
$$

It is easy to see that (5.2)-(5.4) form a closed system. With the boundary conditions that all the velocities vanish at the boundary, the system (5.2)-(5.4) determines the evolutions of the mean flow and the oblique waves both in time and space from a group of initial conditions.

A simplification can be made based on the continuity equation $\nabla \cdot \overline{\mathbf{v}}_{0}=0$. Since $\bar{u}_{0}, \bar{v}_{0}, \bar{w}_{0}$ are functions only of $t$ and $y, \nabla \cdot \overline{\mathbf{v}}_{0}=0$ becomes $\frac{\partial \bar{v}_{0}}{\partial y}=0$ so that $\bar{v}_{0}=0$. The $y$ momentum equation in (5.2) for the mean flow becomes $\partial \bar{p}_{0} / \partial y=g(y, t)$, where $g(y, t)$ represents nonlinear terms. This equation can easily be integrated to get $\bar{p}_{0}$. However, no matter what $\bar{p}_{0}$ we have, it never appears in the other equations. That is to say, $\bar{v}_{0}=0$ is an admissible solution to our equation. Therefore, we take $\bar{v}_{0}=0$ in the following.

Two properties of (5.2)-(5.4) are worthy of comment. First, in these equations the total mean flow term always appears in the form $\bar{U}=U+\bar{u}_{0}$, and there is never any approximation regarding the order of magnitude of $\bar{u}_{0}$ alone. Although previously it was assumed that $\bar{u}_{0} \sim \varepsilon$, the fact that this term always appears in the form $\bar{U}=U+\bar{u}_{0}$ makes this assumption unnecessary. Our equations will be a good approximation of the real dynamic equations as long as $\bar{w}_{0}, \mathbf{v}_{0}, p_{0}, \mathbf{v}_{+}, p_{+}, \mathbf{v}_{-}$, and $p_{-}$remain small. However, initially $\bar{u}_{0}$ has to be small since the instability of the plane Poiseuille is to be studied. Physically speaking, the release of the restriction on the order of magnitude for $\bar{u}_{0}$ is appropriate because, as noted earlier, this term should change appreciably during the transition process while others should remain small. In the later computations of these equations, it is found that the above statement is indeed true.

Second, it is seen that the expansion forms of the velocity and pressure did not include any terms proportional to $e^{2 i \alpha x+2 i \beta z}$. The underlying assumption is that, initially, this mode does not have any energy. Although during the interactions the nonlinear terms in the dynamic equations may act as an energy provider to make this mode grow, the fact that these nonlinear terms have the order $\varepsilon^{2}$ makes it very unlikely that this mode will grow beyond the same order $\varepsilon^{2}$. Therefore, for example, in the dynamic equations for the primary wave that is proportional to $e^{i \alpha x+i \beta}$, the nonlinear terms coming from the interaction of mode $e^{2 i \alpha x+2 i \beta z}$ and mode $e^{-i \alpha x-i \beta z}$ will have order $\varepsilon^{3}$ and will be considered negligible.

6. Nonlinear instabilities for plane Poiseuille flow. Equations (5.2)-(5.4) together with initial and boundary conditions provide a nonlinear stability problem. Numerical methods must be used to obtain the solutions. Special precautions are necessary in the computations. Again as in the linear stability case, two independent numerical 
methods are used, namely, Chebyshev tau and Chebyshev collocation methods, to cross check the accuracy. The results from the discrete time computations are also compared with those from the eigenvalue computations and the agreement between these different methods is excellent. The details of the computational methods, the verification and comparison results, and the initial conditions are described more fully in the Appendix.

As in the linear stability case, the relative energy of a quantity $f$ (a scalar or a vector) is defined to be

$$
E n(f, t)=e n(f, t) / e n(f, 0), \quad e n(f, t)=\int_{-1}^{1} f \cdot f^{*} d y
$$

Also, some initial amplitudes are defined to be

$$
\begin{aligned}
a_{1} & =\sqrt{e n\left(\bar{u}_{0}, 0\right)}, & & a_{2}=\sqrt{\operatorname{en}\left(\bar{w}_{0}, 0\right)}, \\
a_{3} & =\sqrt{e n\left(u_{0}, 0\right)}, & & a_{4}=\sqrt{\operatorname{en}\left(v_{0}, w_{0}, 0\right)}, \\
a_{5} & =\sqrt{e n\left(\mathbf{v}_{+}, \mathbf{v}_{-}, 0\right)} . & &
\end{aligned}
$$

Because of the expansion forms we take for the velocities and pressure, we have the following value for the total initial perturbation energy $\mathcal{E}_{0}$ :

$\mathcal{E}_{0}=\frac{15}{8} \int_{-1}^{1} \bar{u}_{0} U d y+\frac{15}{16} \int_{-1}^{1}\left(\bar{u}_{0}^{2}+\bar{w}_{0}^{2}\right) d y+\frac{15}{8} \int_{-1}^{1} \mathbf{v}_{0} \cdot \mathbf{v}_{0}^{*} d y+\frac{15}{8} \int_{-1}^{1}\left(\mathbf{v}_{+} \cdot \mathbf{v}_{+}^{*}+\mathbf{v}_{-} \cdot \mathbf{v}_{-}^{*}\right) d y$,

where factors $15 / 8$ and $15 / 16$ come from normalization with respect to that of the basic flow $U$.

We have no intention of investigating all the possible choices of wave number and initial amplitude. Only a few cases are selected for the computations. In this section, (5.2)-(5.4) will be solved by the method described in the Appendix with a pair of wave numbers $\alpha, \beta$, and a set of the corresponding unknowns $\overline{\mathbf{v}}_{0}, \mathbf{v}_{0}, \mathbf{v}_{+}$, and $\mathbf{v}_{-}$. The truncation errors will be discussed at the end of the section.

In the first computation, the parameters and the initial conditions are as follows:

\begin{tabular}{|llllll}
$R=1200$, & $\mathcal{E}_{0} \doteq 0.0015$, & $\alpha=1$, & $\beta=2$, & $k_{1}=5$, & $k_{2}=3$, \\
$a_{1}=0.015$, & $a_{2}=0.015$, & $a_{3}=0.015$, & $a_{4}=0.01$, & $a_{5}=0.015$. & \\
\hline
\end{tabular}

In Figures 5 and 6 , we plot the initial profiles of $u_{0}$ and $u_{+}$. The methods to compute these initial values are fully explained in the Appendix. Generally speaking, they are the least stable or most unstable eigenfunctions of the quasilinear eigenvalue problems derived from (5.3) and (5.4) by neglecting all or certain forcing terms in the equations. Figure 7 shows the evolution of all kinds of energies with time. Figure 8 presents contour plots of the instantaneous $x$-direction velocity in the $(y, z)$-plane at $t=0,140,160$, and 300 , where $0,1(0.1)$ means the contour values are plotted from 0 to 1 in intervals of 0.1 . Figure 9 shows the evolution of the $x$-direction mean velocity profile at different times.

Overall, there are subcritical instabilities and neutral stabilities as well as some oscillations and decays. The striking feature in the energy plot is that all the energies undergo a jump except that of $u_{0}$ around $t=160$. It is seen that around this point the status of the total mean flow and the three-dimensional waves suddenly changes. 


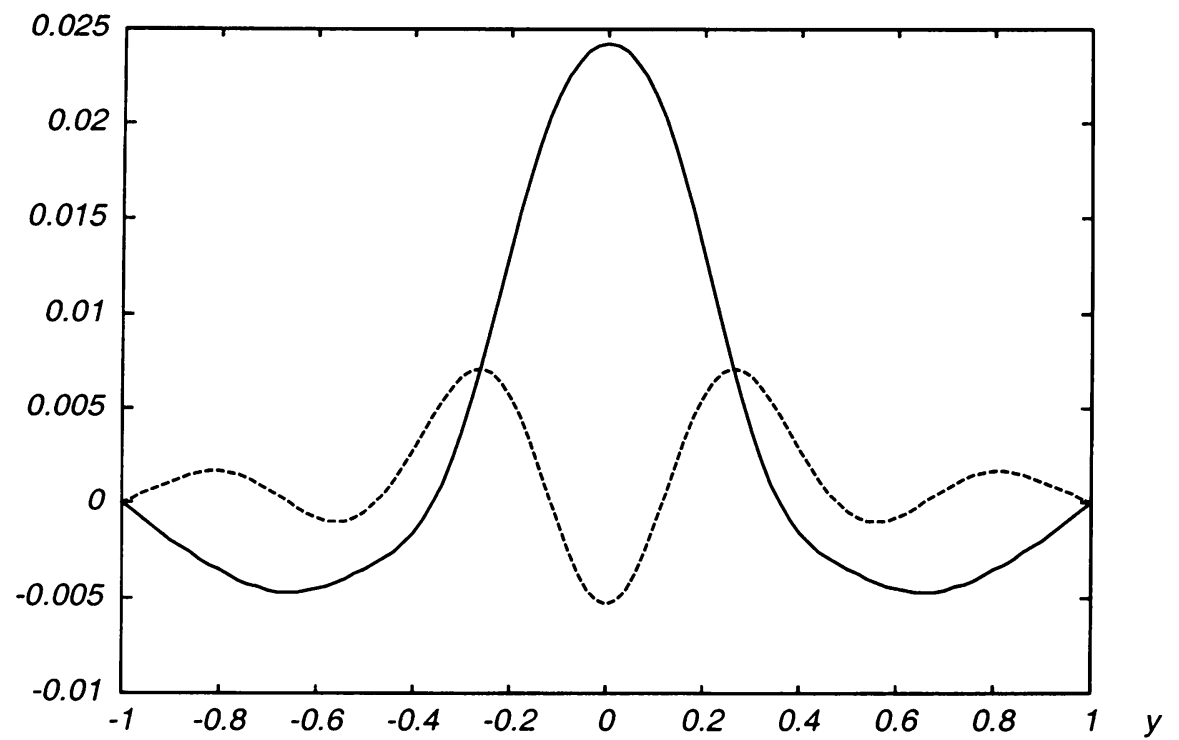

FIG. 5. A plot of the initial $u_{0}$ in the first computation. Solid line: real part. Dashed line: imaginary part. $R=1200, \alpha=1, \beta=$ $2, a_{1}=a_{2}=a_{3}=a_{5}=0.015, a_{4}=0.01, k_{1}=5, k_{2}=3$.

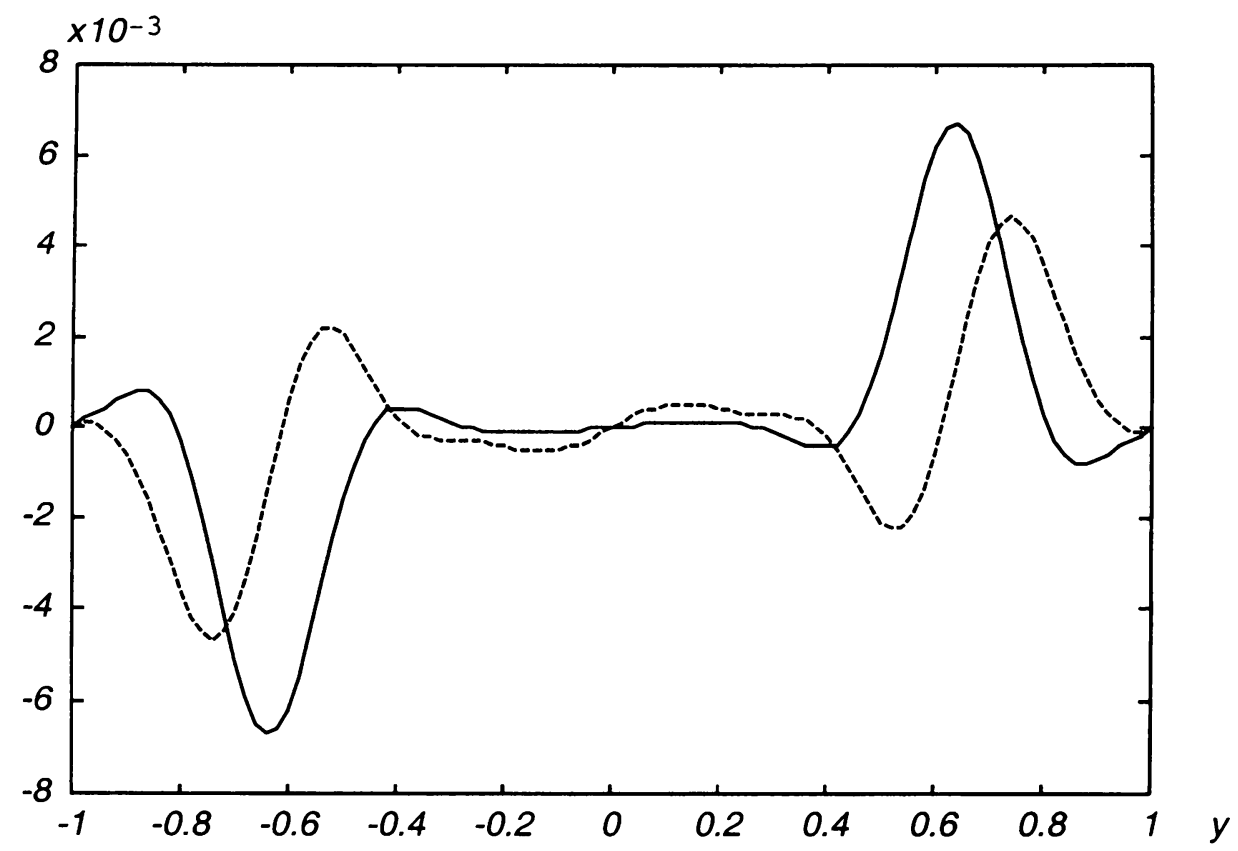

FIG. 6. A plot of the initial $u_{+}$in the first computation. Solid line: real part. Dashed line: imaginary part. 


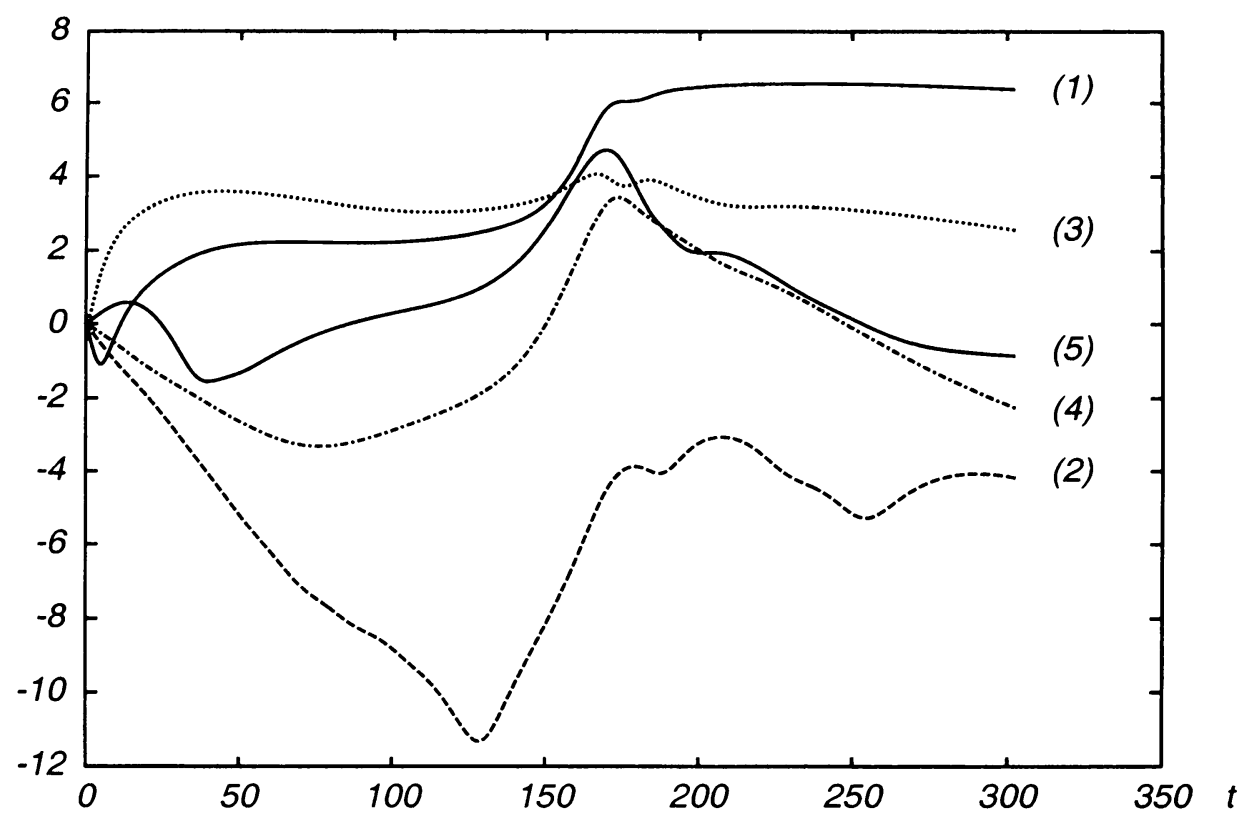

FIG. 7. Energy evolutions in the first computation. (1): $\log \left[\operatorname{En}\left(\bar{u}_{0}, t\right)\right], \quad(2): \quad \log \left[\operatorname{En}\left(\bar{w}_{0}, t\right)\right], \quad(3): \quad \log \left[\operatorname{En}\left(u_{0}, t\right)\right], \quad(4):$ $\log \left[\operatorname{En}\left(v_{0}, w_{0}, t\right)\right],(5): \log \left[E n\left(\mathbf{v}_{+}, \mathbf{v}_{-}, t\right)\right]$.

Roughly speaking, these waves grow before this point but become neutral or decay afterward. The transition time for this change of status is from about $t=140$ to $t=180$. Notice that the real time $t^{*}$ has the relation $t^{*}=t l / U_{c}$ with nondimensional time $t$ here. For large $U_{c}$, as $t$ changes from 140 to 180, the real time $t^{*}$ makes a very short change. Equivalently, the jump of the flow status is accomplished in a very short moment. From the profiles of the $x$-direction mean velocity, $U+\bar{u}_{0}$, at $t=80,110$ and 140 (Figure 9), it is seen that a pair of inflection points is developing during this period. From $t=160$ to $t=200$, the mean flow profile becomes highly inflectional, and this makes the two- and three-dimensional waves strongly unstable. The $x$-direction vorticity in the $(y, z)$-plane is shown in Fig. 10.

In conjunction with these figures, it is not hard to see that there are characteristically different patterns before and after the jump, and these patterns may be seen from two main aspects. First, the mean velocity profile changes from a parabola-like shape before the jump to a profile like what is observed in turbulence after the jump. Second, from Fig. 10, the $x$-direction vorticity $\omega$ has remarkably different typical values before, during, and after the jump. Before the jump, $|\omega(t=0)|$ and $|\omega(t=140)| \sim 0.3$. During the jump, at $t=160$, the typical value for $|\omega|$ is 1.27 . After the jump, large and strong vortices become smaller and weaker, and finally $|\omega| \sim 0.1$ at $t=300$. The occurrence of very strong vorticity during the transition period indicates the collapse of the laminar flow. Also noticeable are the similarities and differences in the shapes of the vortices between $t=140$ and $t=300$ in Fig. 10. It appears that the vortices at $t=140$ have 

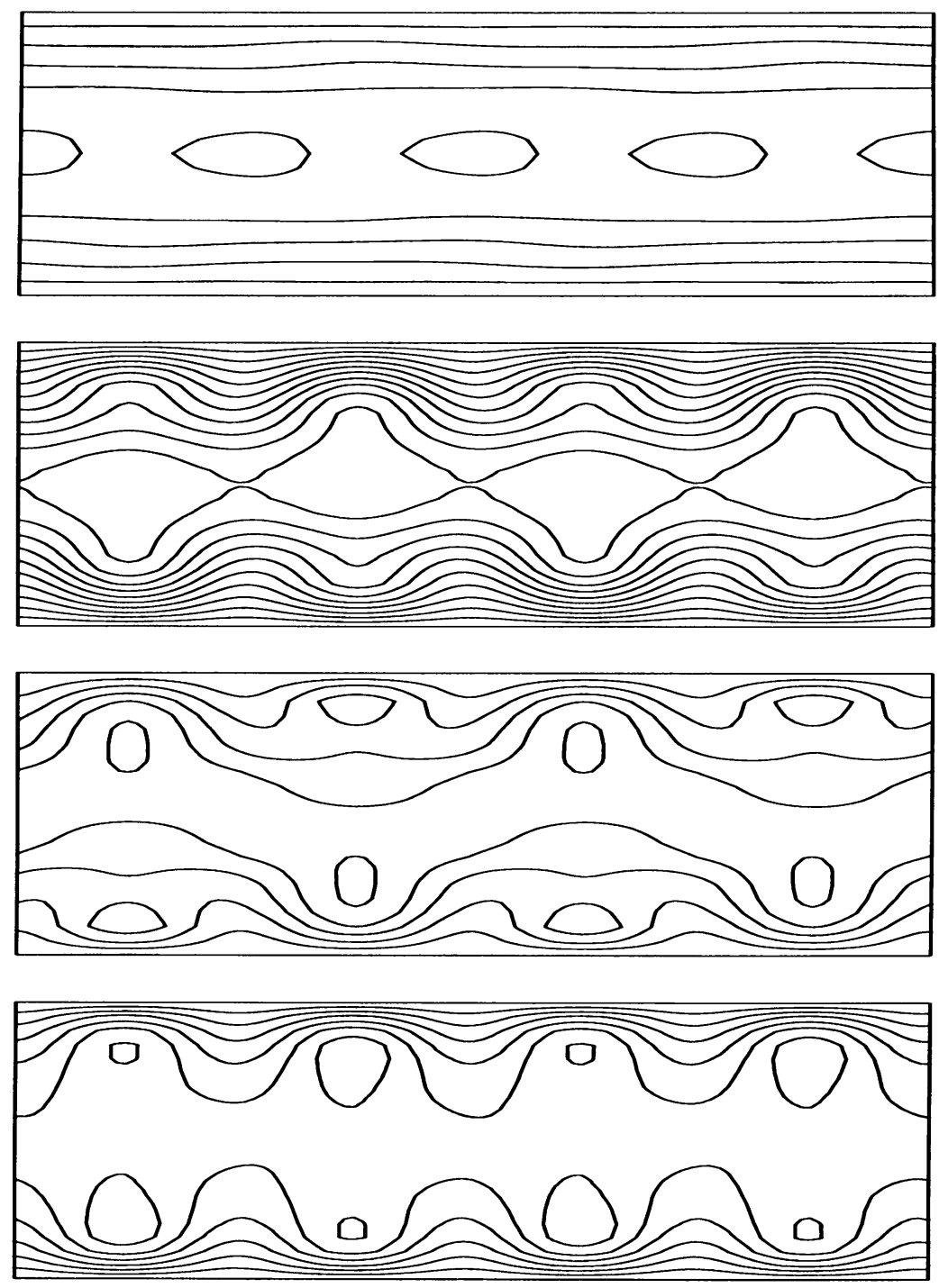

FIG. 8. From top to bottom, contour plots of the $x$-direction velocity in the first computation at $x=0$ and $t=0, t=140, t=160, t=$ $300,0,1(0.1)$. The horizontal lines are $-\pi \leq z \leq \pi$ and the vertical lines are $-1 \leq y \leq 1$.

been broken in half to produce the smaller vortices at $t=300$. Compared with those at $t=0, t=140$, and $t=160$, the vortices at $t=300$ are more uniformly distributed.

These vorticity changes are believed to be consistent with the general experimental observation on turbulent flow, which states that bigger vortices break into smaller ones during the transition process. The changes in the profile of the mean flow velocity, $U+\bar{u}_{0}$ in Fig. 9, are also believed to be consistent with the observations from the experiments. For comparison, the mean flow profiles found in the experiment on the 

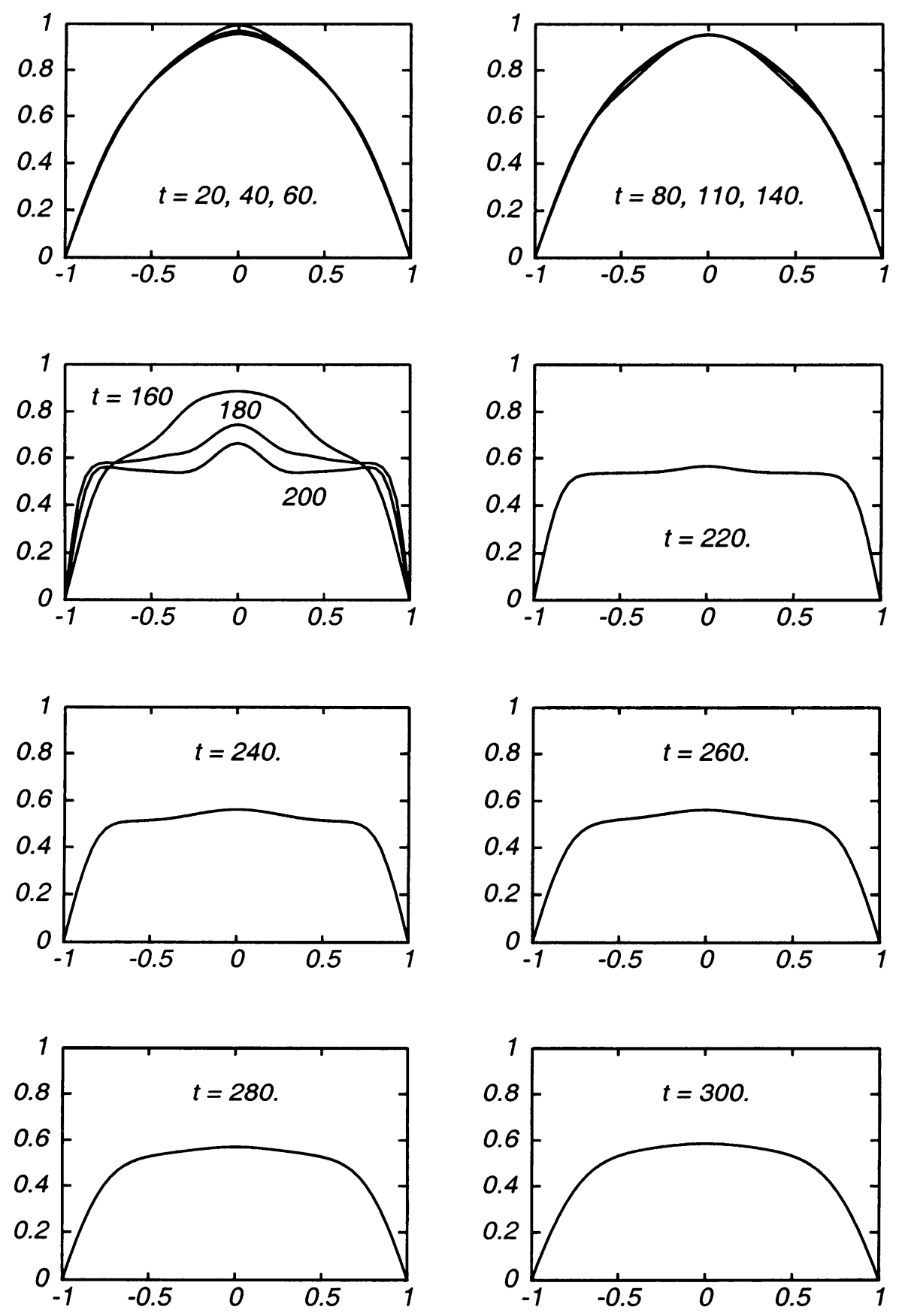

FIG. 9. Evolutions of the $x$-direction mean velocity $U+\bar{u}_{0}$ in the first computation. The horizontal lines are $-1 \leq y \leq 1$. 

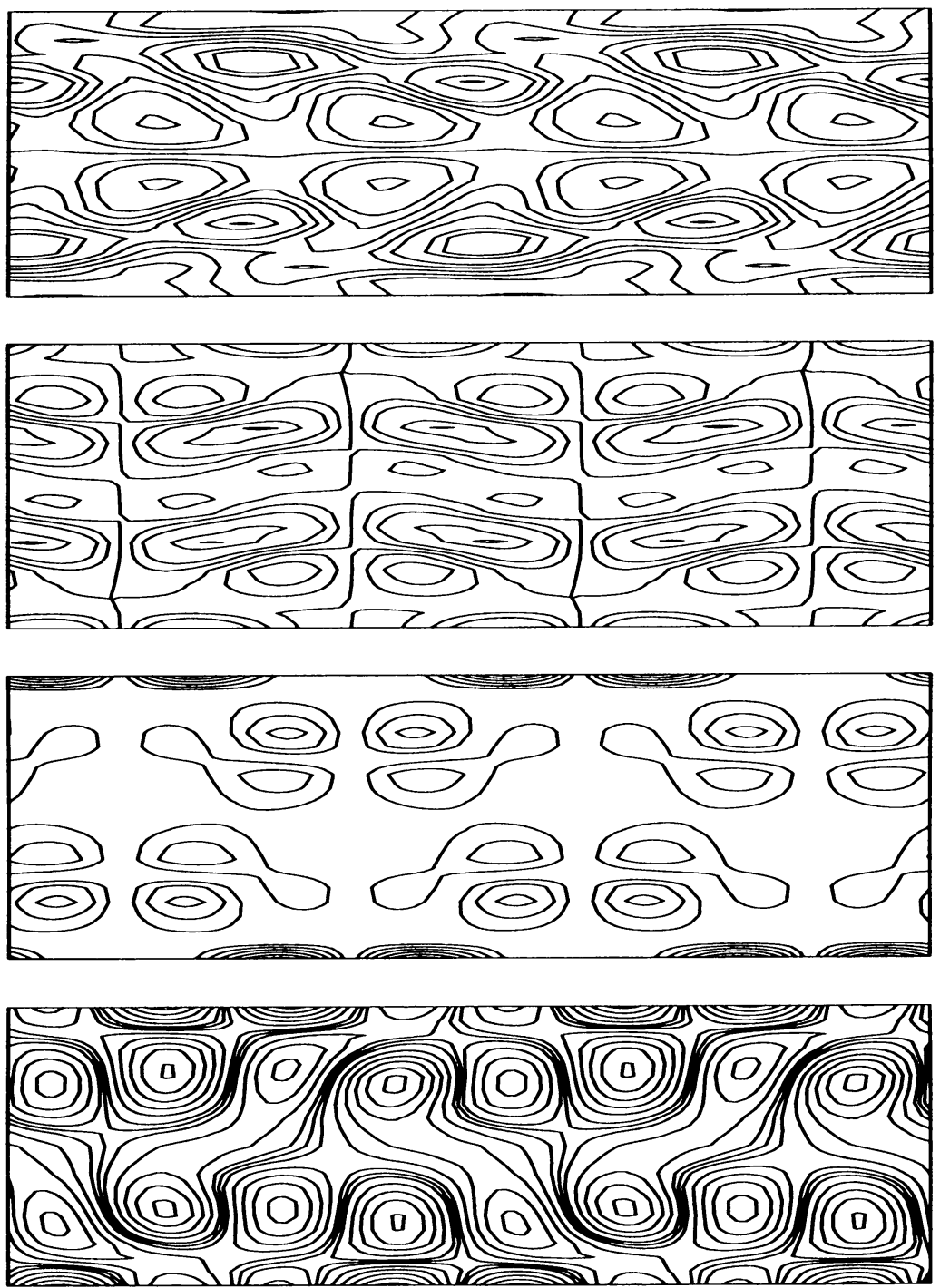

Fic. 10. From top to bottom. contour plots of the $x$-direction vorticity in the first computation at $x=0, t=0,-0.3 .0 .3(0.05)$ : $t=140,-0.3,0.3(0.05): t=160,-1.2,1.2(0.2) ; t=300,-0.1 .0 .1$ (0.02). The horizontal lines are $-\pi \leq z \leq \pi$ and the vertical lines are $-1 \leq y \leq 1 .|\omega(t=0)| \sim 0.3 .|\omega(t=140)| \sim 0.3,|\omega(t=160)| \sim$ $1.27,|\omega(t=300)| \sim 0.1$. 


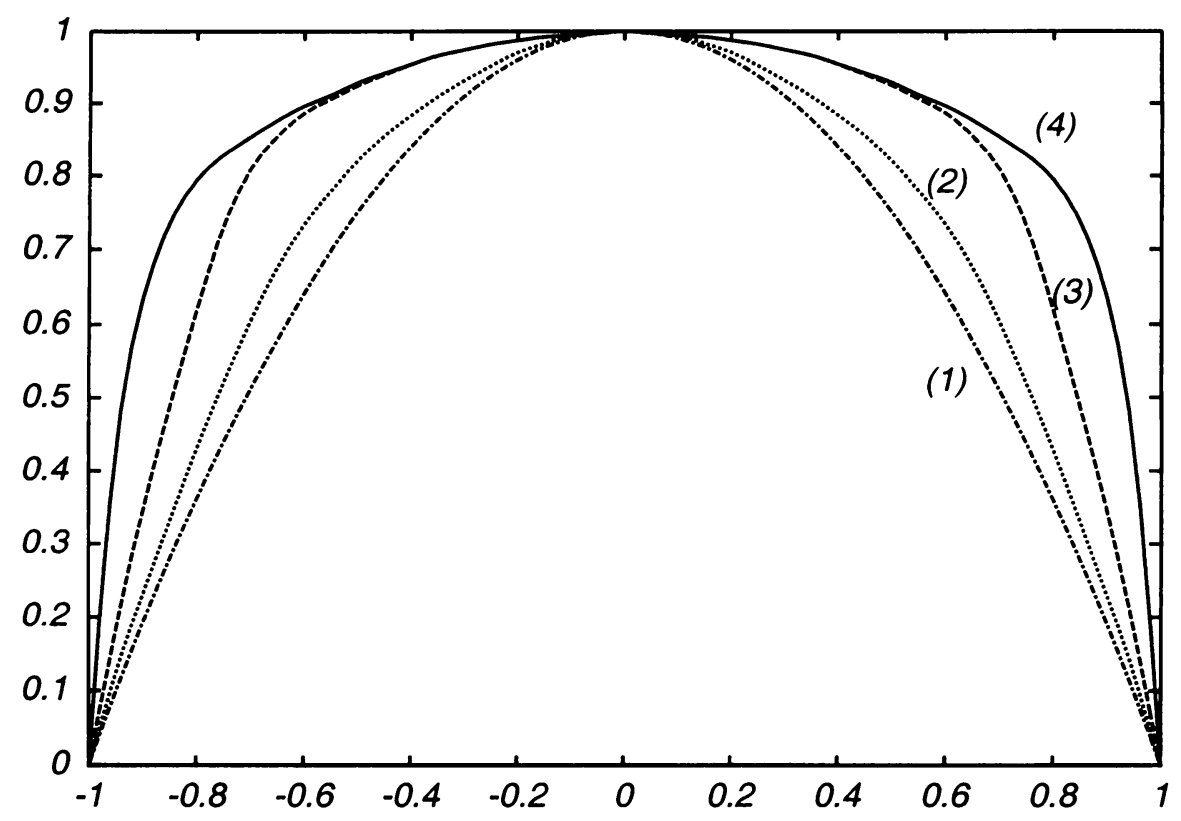

FIG. 11. The nondimensional mean flow velocity profile $U / U_{c}$ from the experiment by M. Nishioka and M. Asai [24]. (1): $R=1010,(2)$ : $R=1150,(3): R=1600,(4): R=3950$.

subcritical transition in plane Poiseuille flow by M. Nishioka and M. Asai [24] are cited here in Fig. 11. In this experiment, the disturbance introduced into the flow is of high intensity, $10-20 \%$ of $U_{c}$, where $U_{c}$ is the centerline velocity of the basic flow. As $R$ increases, the mean velocity profile becomes more and more flat around the centerline and appears to be moving towards the mean flow profile in turbulence. In Fig. 12, the nondimensional mean velocity profile in Fig. 9 at $t=300$ is plotted against that of $R=1600$ from the above experiment. It is easy to see that these two profiles are indeed very similar.

There are several new features in the results from the present analysis that are different from the results of the previous theories. First, subcritical instabilities are obtained directly from the multiple wave interactions. In this approach, there is no pre-existing condition such as the formation of a secondary flow in plane Poiseuille flow by Orszag and Patera [14]. Second, the evolutions of the mean flow profile (Fig. 9) indicate that an inflectional and afterwards irregular mean flow profile may be the decisive factor causing the small amplitude oblique waves to become unstable. At this point, it is worthwhile to compare our results with those from the direct numerical simulation by Orszag and Kells [16]. In the work by Orszag and Kells, where perturbations with much larger initial amplitude were used, the evolution of the mean flow profile did not show any 


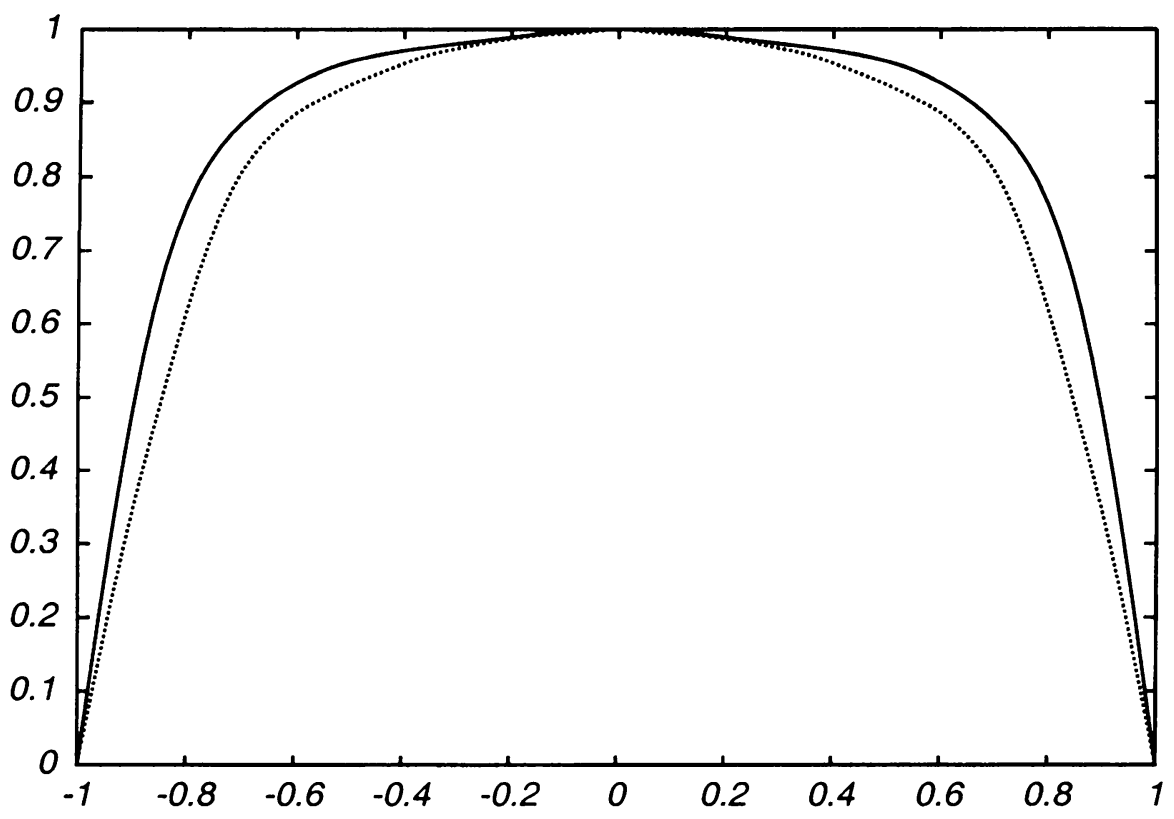

FIG. 12. Solid line: the nondimensional mean flow velocity profile $U / U_{c}$ of Fig. 9 at $t=300$. Dotted line: the curve (3) in Fig. 11.

appreciable inflection point. Although strong inflections were found in the instantaneous $x$-direction velocity profiles, these inflections come mainly from the contributions of the perturbation waves. When the amplitudes of the disturbances are not large enough to give sufficient contributions, these inflections in the instantaneous $x$-direction velocity may disappear or become very weak so that the total flow may become stable to threedimensional waves. It is believed that this explains why no instabilities were found for smaller amplitude perturbation waves in their computation. On the contrary, the mean flow profile in the present analysis becomes very inflectional and thus makes it possible for small amplitude disturbances to become unstable. Third, the jump phenomenon of the perturbation waves is found in the present analysis. While large perturbations can directly lead to transition, we speculate that for small amplitude perturbations like those in the present computation, the transition starts with the jump. The jump phenomenon will be discussed later in this section after more information has been obtained through further computations.

The second computation is for a higher Reynolds number, $R=2000$, with the parameters and the amplitudes in the following table:

\begin{tabular}{|llllll|}
\hline$R=2000$, & $\mathcal{E}_{0} \doteq 0.00078$, & $\alpha=1$, & $\beta=2$, & $k_{1}=5$, & $k_{2}=3$, \\
$a_{1}=0.01$, & $a_{2}=0.01$, & $a_{3}=0.01$, & $a_{4}=0.01$, & $a_{5}=0.01$. & \\
\hline
\end{tabular}




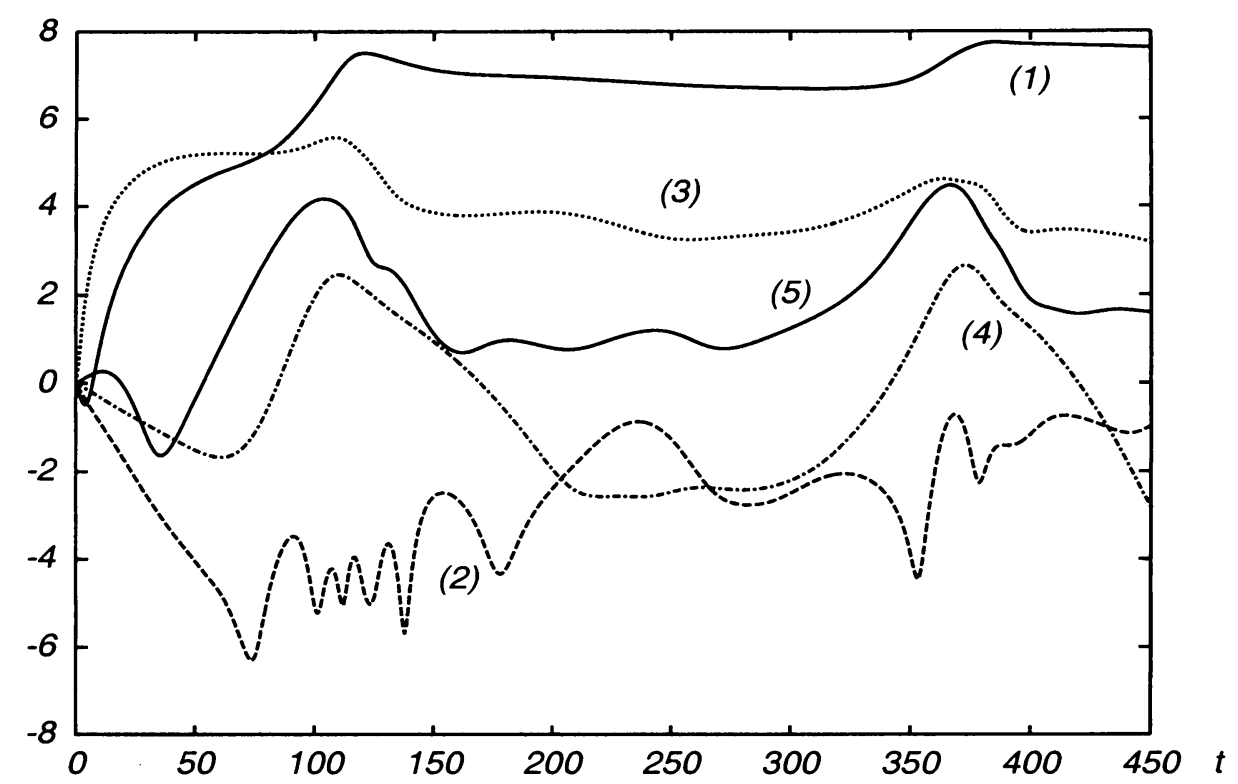

FIG. 13. Energy evolutions in the second computation at $R=2000$, $\alpha=1, \beta=2, a_{1}=a_{2}=a_{3}=a_{4}=a_{5}=0.01, k_{1}=5, k_{2}=$ 3. (1): $\log \left[\operatorname{En}\left(\bar{u}_{0}, t\right)\right],(2): \log \left[\operatorname{En}\left(\bar{w}_{0}, t\right)\right],(3): \log \left[\operatorname{En}\left(u_{0}, t\right)\right]$, (4): $\log \left[E n\left(v_{0}, w_{0}, t\right)\right],(5): \log \left[E n\left(\mathbf{v}_{+}, \mathbf{v}_{-}, t\right)\right]$.

The initial perturbation energy in this computation is about half of that used in the $R=1200$ case, and the evolutions are traced for a longer time. The purpose of this computation is to investigate the instability behavior of the flow of a larger $R$ but with smaller initial perturbations. Figure 13 shows the evolutions of the wave energy, and in Figs. 14 and 15, the profiles of the $x$-direction mean flow $U+\bar{u}_{0}$ are plotted.

In this case, it is a little surprising that two jumps are found in the evolution of the flow quantities. The first one is from $t=100$ to $t=130$, and the second one is between $t=350$ and $t=400$. During these jumps, the energies of the oblique wave increase rapidly, and the mean flow velocity profiles have inflection points and take very irregular shapes. After these jumps, the energies of the oblique waves change slowly, and the mean flow profiles become smooth and regular. In addition, the mean flow profile after the second jump is more flat and is more like the mean velocity profile observed in turbulence than it was after the first jump. The $x$-direction vorticity $\omega$ profiles are plotted in Figs. 16 and 17 at $x=0$ and $z=2 \pi / 5$, where the maximum values of $\omega$ occur. For Figs. 16 and 17 , two times are chosen during the two jumps, and another two times are chosen after the jumps. Figures 16 and 17 show that during these jumps, for example, at $t=120$ and $t=360$, strong vorticities are produced and the highest intensity is near boundaries $y= \pm 1$. After the jumps, for instance, at $t=240$ and $t=450$, the vorticities become much weaker and have a more even distribution across the flow domain. 

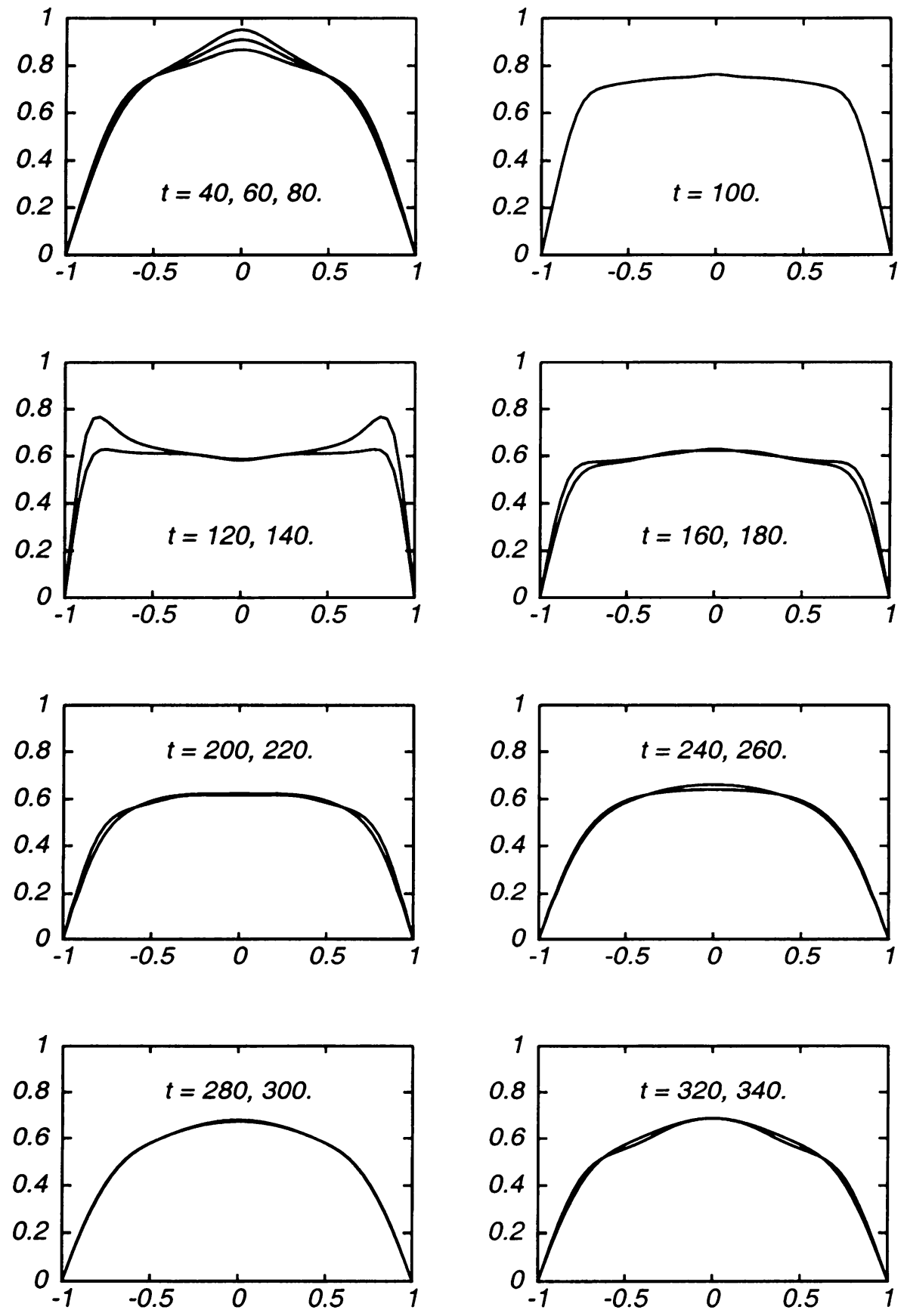

FIG. 14. Evolutions of the $x$-direction mean velocity $U+\bar{u}_{0}$ in the second computation $(R=2000)$. The horizontal lines are $-1 \leq y \leq 1$. 

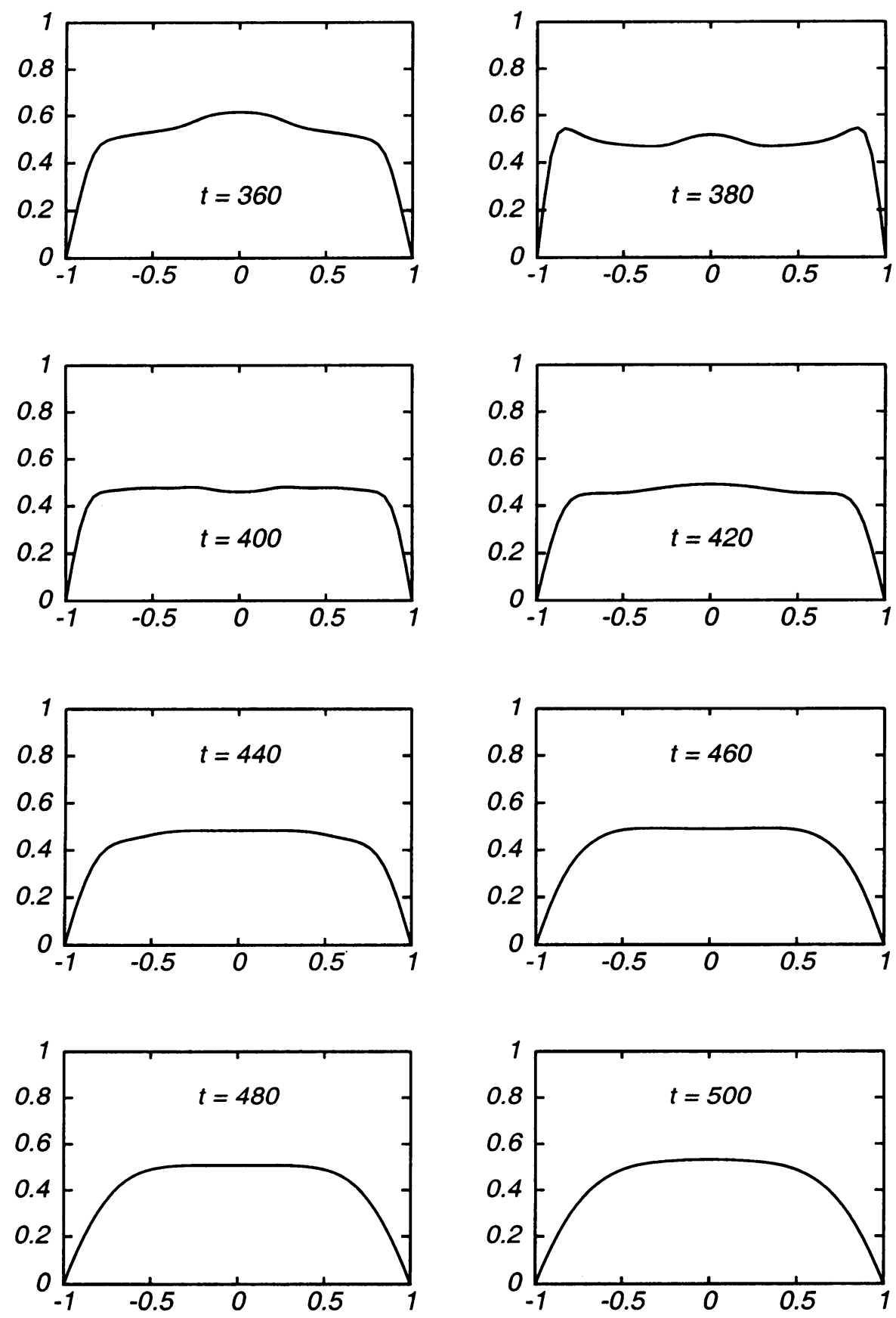

FIG. 15. Evolutions of the $x$-direction mean velocity $U+\bar{u}_{0}$ in the second computation $(R=2000)$. The horizontal lines are $-1 \leq y \leq 1$. 


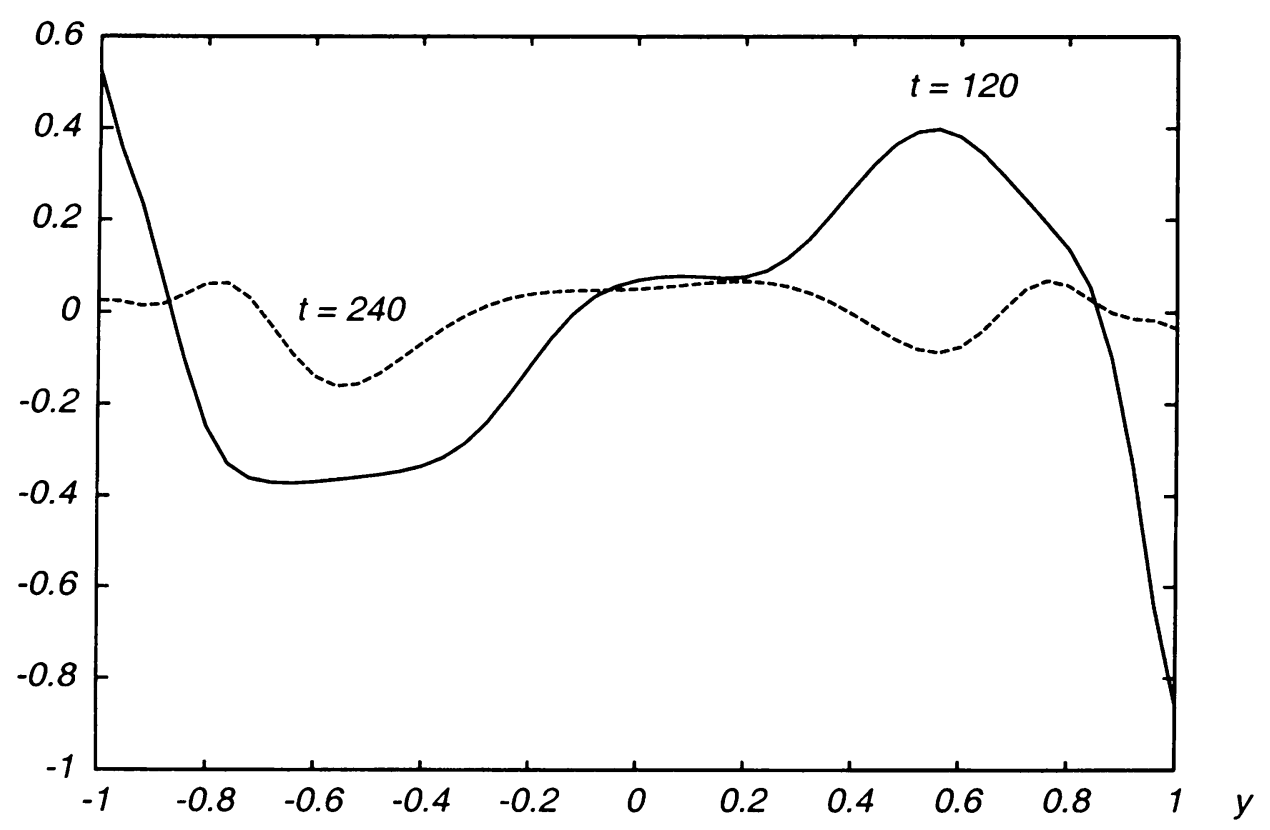

FIci. 16. A plot of the $x$-direction vorticity of the flow at $x=0, z=$ $2 \pi / 5, t=120$ and $t=240$ in the second computation $(R=2000)$.

Taking into consideration the results from the first and the second computations, we believe that these jumps are associated with the intermittent character of the disturbance observed in the transition experiment. The flow patterns shown in Figs. 13 through 17 are very similar to the description of the intermittency phenomenon first observed by Reynolds [25], which is cited here:

another phenomena... was the intermittent character of the disturbance. The disturbance would suddenly come on through a certain length of tube and pass away and then come on again, giving the appearance of flashes, and these flashes would often commence successively at one point in the pipe. The appearance when the flashes succeded each other rapidly was as shown.

The successive appearance of the irregular mean flow profiles and the strong disturbances seen in Figs. 13 through 17 resemble closely the appearance of the flashes observed by Reynolds. These features indicate that the physical process has been correctly captured by the approach and also suggest that the transition processes are different for smallamplitude disturbances and for large-amplitude disturbances. In the former case, transition begins at the appearance of the intermittent character of the disturbance, or from the energy jumps of the disturbance. In the latter case, the intermittence phenomenon can be bypassed, as shown by the work of Orszag and Kells [16].

For lower Reynolds numbers, the case $R=1000$ is computed, with the wave numbers and the initial amplitudes the same as those in the first computation. The parameters 


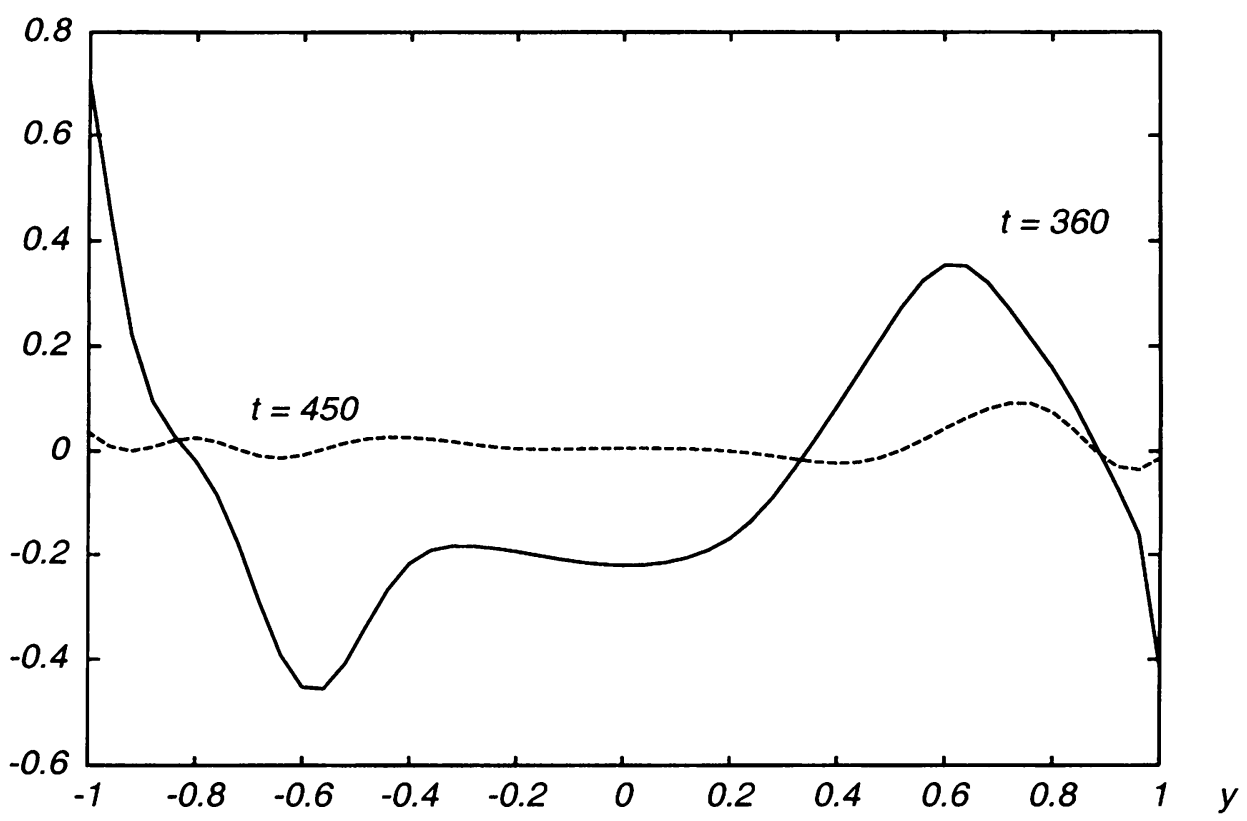

FIG. 17. A plot of the $x$-direction vorticity of the flow at $x=0, z=$ $2 \pi / 5, t=360$ and $t=450$ in the second computation $(R=2000)$.

and the initial conditions are in the table below:

\begin{tabular}{|llllll}
\hline$R=1000$, & $\mathcal{E}_{0} \doteq 0.0015$, & $\alpha=1$, & $\beta=2$, & $k_{1}=5$, & $k_{2}=3$, \\
$a_{1}=0.015$, & $a_{2}=0.015$, & $a_{3}=0.015$, & $a_{4}=0.01$, & $a_{5}=0.015$. & \\
\hline
\end{tabular}

The purpose of this computation is to attain knowledge about the so-called critical Reynolds number, which is believed to be a function of the wave numbers and the initial conditions. Although there is no intention to seek such a critical $R$ in all the possible cases, we believe, nevertheless, that some information can be obtained by these examples.

In Figs. 18 and 19, energy curves and the mean flow velocity profiles are plotted. Contrary to the results in the $R=1200$ case, at $R=1000$, there is no jump in the energy curves, and everything damps after a short period of time. The mean velocity profiles do not change much from the perfect parabola. These totally different flow characteristics between $R=1200$ and $R=1000$ give clear evidence of the existence of a critical Reynolds number, which is about $R=1000$ in this particular case.

There is no restriction in this theory on the number of the waves that can participate in the interactions, so long as these waves do not form resonant triads. Two sets of the two- and the three-dimensional waves that participated in the interactions cases are also computed for both $R=1000$ and $R=2000$, and the results are similar to the results presented above and therefore will not be reported here. 


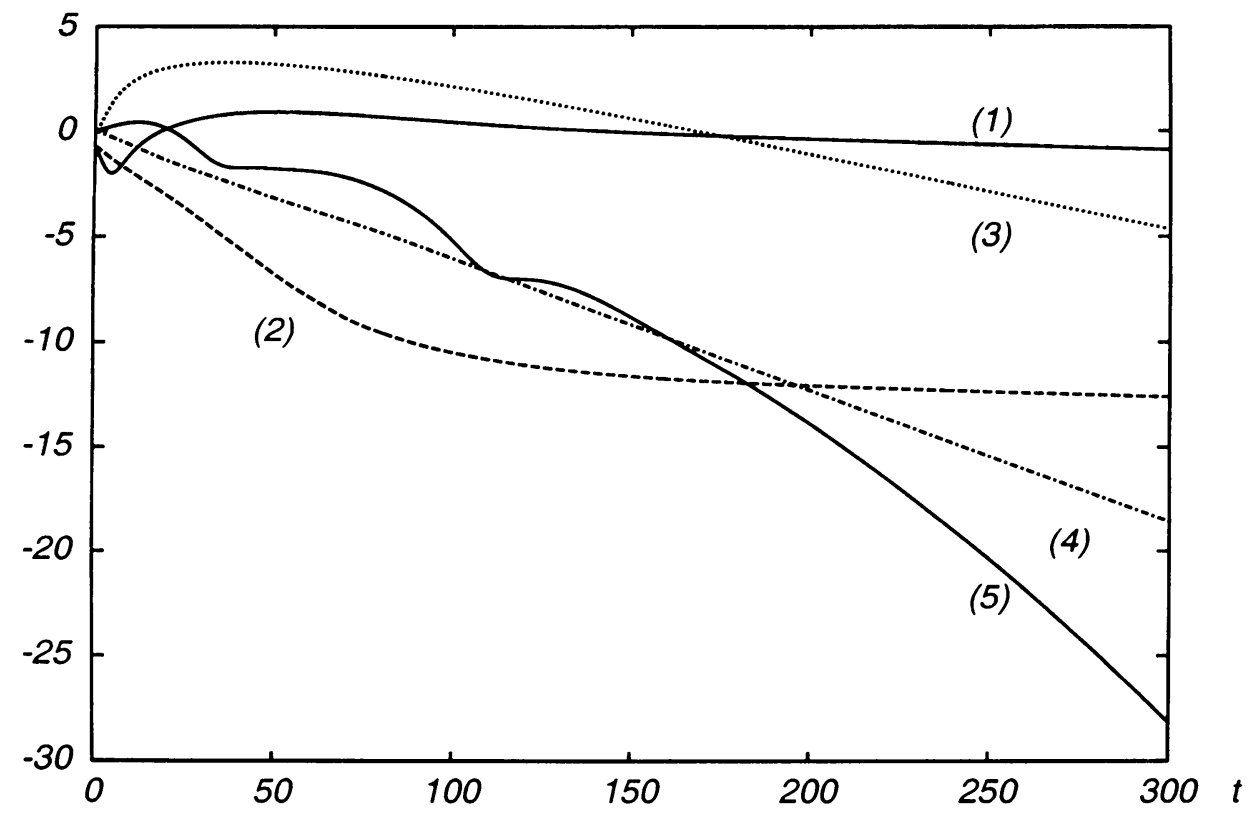

FIG. 18. Energy evolutions of the third computation at $R=1000$, $\alpha=1, \beta=2, a_{1}=a_{2}=a_{3}=a_{5}=0.015, a_{4}=0.01, k_{1}=5, k_{2}=$ 3. (1): $\log \left[\operatorname{En}\left(\bar{u}_{0}, t\right)\right],(2): \log \left[\operatorname{En}\left(\bar{w}_{0}, t\right)\right],(3): \log \left[\operatorname{En}\left(u_{0}, t\right)\right],(4):$ $\log \left[E n\left(v_{0}, w_{0}, t\right)\right],(5): \log \left[E n\left(\mathbf{v}_{+}, \mathbf{v}_{-}, t\right)\right]$.

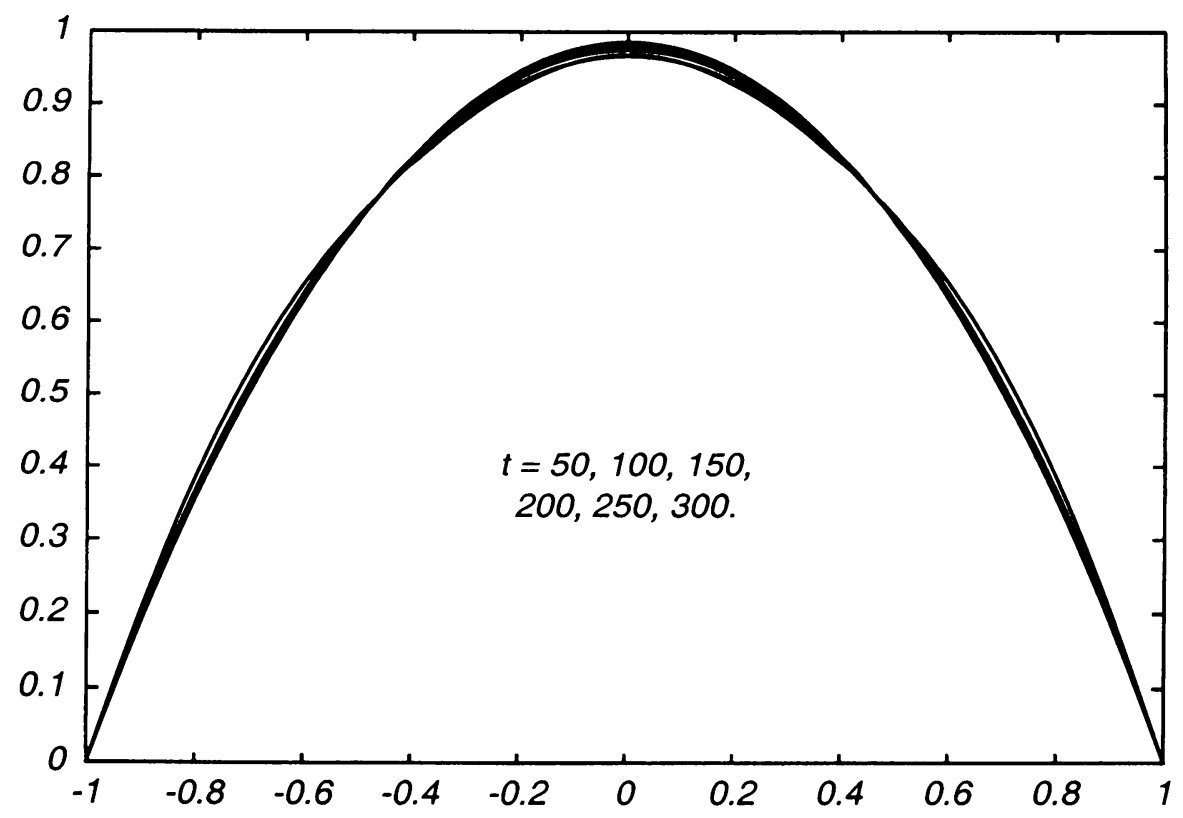

FIG. 19. Evolutions of the $x$-direction mean velocity $U+\bar{u}_{0}$ in the third computation $(R=1000)$. 
To end this section, we discuss the truncation errors. As was stated in the derivation of the equations, if we assume $\bar{w}_{0}, \mathbf{v}_{0}, \mathbf{v}_{+}, \mathbf{v}_{-}, \sim \varepsilon$, our equations are accurate to $\varepsilon^{2}$, since $\varepsilon$ and $\varepsilon^{2}$ terms are kept in the equations, with $\varepsilon^{3}$ terms neglected. Moreover, the amplitude of $\bar{u}_{0}$ will not affect the accuracy of the equations because no approximation was made with respect to it. Now, from the energy curves in Fig. 7, we see that except for that of $\bar{u}_{0}$, the most amplified energy is for $\left(\mathbf{v}_{+}, \mathbf{v}_{-}\right)$and $\max \left\{\log \left[\operatorname{En}\left(\mathbf{v}_{+}, \mathbf{v}_{-}, t\right)\right]\right\}=4.7$.

Similarly, for energy curves in Fig. 13, it is found that $\max \left\{\log \left[\operatorname{En}\left(u_{0}, t\right)\right]\right\}=5.57$. Using the notation

$$
\varepsilon_{1}=\max \left\{\sqrt{e n\left(\mathbf{v}_{+}, \mathbf{v}_{-}, t\right)}\right\}, \quad \varepsilon_{2}=\max \left\{\sqrt{e n\left(u_{0}, t\right)}\right\},
$$

the table below indicates the truncation errors at these most amplified points:

$$
\begin{array}{|llll}
\hline \varepsilon_{1}=0.157, & \varepsilon_{1}^{2}=0.0246, & \varepsilon_{1}^{3}=0.00387, & \frac{\varepsilon_{1}^{3}}{\varepsilon_{1}+\varepsilon_{1}^{2}} \doteq 0.02 \\
\varepsilon_{2}=0.162, & \varepsilon_{2}^{2}=0.0262, & \varepsilon_{2}^{3}=0.00425, & \frac{\varepsilon_{2}^{3}}{\varepsilon_{2}+\varepsilon_{2}^{2}} \doteq 0.023
\end{array}
$$

Therefore, even in these worst-case situations, we believe that our results are at least qualitatively correct.

7. Conclusion. The application of the multiple wave interactions at the initial presence of a small mean flow perturbation term indicates that several important properties of the plane Poiseuille flow instability process can be predicted by this theory. In the linear theory part, it is shown that the original plane Poiseuille flow is sensitive to small alterations to the mean flow profile. With a small mean flow alteration, the critical Reynolds number can be shifted well below 1000. In the nonlinear theory part, when the nonlinear interactions take place, the total mean flow velocity $U+\bar{u}_{0}$ profile changes rapidly toward the mean flow profile observed in turbulence while other oblique wave quantities oscillate but remain small. Although the mean velocity profiles obtained are not to be interpreted as mean turbulent velocity profiles, their tendencies, the jumps of the flow quantities, and especially the characteristically different vorticity patterns during and after the jumps, lead us to speculate that this theory correctly captures some of the properties pertaining to the transition process.

Based on the results and analysis, some projections on the plane Poiseuille flow subcritical transition process in the initially small amplitude disturbance situation can be proposed. We believe that in this situation, the transition to turbulence begins with the interaction of multiple perturbation waves, especially the interaction between the mean flow and the oblique waves. The fact that the mean perturbation term is an exact solution of the Navier-Stokes equations indicates that this term can directly come from the flow field as a result of some disturbances in the flow. The amplitude of this term can be arbitrary, and this makes it possible for it to appear in the interaction as a more powerful partner. At initial times, the appearance of the small mean perturbation is crucial for the growth of the oblique waves. This initial growth makes it possible for these oblique waves to become strong and then to play their roles in the nonlinear interaction. The entire transition process may contain several parts, depending on the initial intensity of the disturbance. During each of these parts, the oblique waves undergo a rapid change and 
a slow change period, and the mean flow profile repeats an irregular and regular shape, giving rise to the intermittence phenomenon in the experiment. By repeating the irregular and regular shape, the mean flow profile changes its spatial configuration towards the mean velocity profile observed in turbulent flows. For large initial disturbance cases, the entire transition process may contain only one or two of the above-mentioned parts, or it may be that these parts are so close to each other that they are not distinguishable in the expcriment.

Finally, though the derivation, the demonstration, and the application have been made only for the plane Poiseuille flow case, the theory is applicable to other parallel flow situations. In fact, the linear and nonlinear stability investigations were also carried out in the plane Couette flow case and similar results were obtained.

Acknowledgment. I wish to express my thanks to Professor D. J. Benney in the Mathematics Department at MIT, for his guidance, encouragement and stimulating discussions. The K. C. Wong Education Foundation provided a scholarship for my first two years at MIT, where the research in this paper began. I sincerely thank this Foundation for its help. My thanks also go to the referees of this paper for their careful reading and valuable suggestions and comments.

Appendix. In this appendix, the computational methods, the verification and the comparison of the results from different numerical methods, and the initial conditions of (5.2)-(5.4) are discussed.

Numerical method. For convenience, we use the notation

$$
\mathbf{v}_{1}=\mathbf{v}_{+}+\mathbf{v}_{-}, \quad p_{1}=\left(p_{+}, p_{-}\right)
$$

Then the equations (5.2)-(5.4) can be rewritten as

$$
\begin{aligned}
& \frac{\partial \overline{\mathbf{v}}_{0}}{\partial t}=\bar{D}_{0} \overline{\mathbf{v}}_{0}+\bar{G}_{0}, \\
& \frac{\partial \mathbf{v}_{0}}{\partial t}=D_{0} \mathbf{v}_{0}+E_{0} p_{0}+G_{0}, \\
& \frac{\partial \mathbf{v}_{1}}{\partial t}=D_{1} \mathbf{v}_{1}+E_{1} p_{1}, \\
& \nabla \cdot \mathbf{v}_{0}=0, \quad \nabla \cdot \mathbf{v}_{1}=0,
\end{aligned}
$$

where $\bar{D}_{0}, D_{0}, D_{1}, \bar{G}_{0}, G_{0}, E_{0}$, and $E_{1}$ are matrices representing corresponding differential operators in the equations and are functions of $y, \overline{\mathbf{v}}_{0}, \mathbf{v}_{0}$, and $\mathbf{v}_{1}$. The boundary conditions on (A.1) are

$$
\overline{\mathbf{v}}_{0}(y= \pm 1, t)=0, \quad \mathbf{v}_{0}(y= \pm 1, z, t)=0, \quad \mathbf{v}_{1}(x, y= \pm 1, z, t)=0
$$


The time advancement is carried out by the Crank-Nicholson scheme, and a complete time step consists of the following three steps:

$$
\begin{aligned}
& \frac{1}{\Delta t}\left(\overline{\mathbf{v}}_{0}^{n+1}-\overline{\mathbf{v}}_{0}^{n}\right)=\frac{1}{2}\left(\bar{D} \overline{\mathbf{v}}_{0}^{n+1}+\bar{D} \overline{\mathbf{v}}_{0}^{n}\right)+\bar{G}_{0}\left(\mathbf{v}_{0}^{n}, \mathbf{v}_{1}^{n}\right), \quad \nabla \cdot \overline{\mathbf{v}}_{0}^{n+1}=0, \\
& \frac{1}{\Delta t}\left(\mathbf{v}_{0}^{n+1}-\mathbf{v}_{0}^{n}\right)=\frac{1}{2}\left(D_{0}^{n+1} \mathbf{v}_{0}^{n+1}+D_{0}^{n} \mathbf{v}_{0}^{n}\right)+\frac{1}{2}\left(E_{0} p_{0}^{n+1}+E_{0} p_{0}^{n}\right)+G_{0}\left(\mathbf{v}_{1}^{n}\right), \quad \nabla \cdot \mathbf{v}_{0}^{n+1}=0, \\
& \frac{1}{\Delta t}\left(\mathbf{v}_{1}^{n+1}-\mathbf{v}_{1}^{n}\right)=\frac{1}{2}\left(D_{1}^{n+1} \mathbf{v}_{1}^{n+1}+D_{1}^{n} \mathbf{v}_{1}^{n}\right)+\frac{1}{2}\left(E_{1} p_{1}^{n+1}+E_{1} p_{1}^{n}\right), \quad \nabla \cdot \mathbf{v}_{1}^{n+1}=0, \quad(\mathrm{~A} .4)
\end{aligned}
$$

with the boundary conditions imposed on the velocities at $t^{n+1}=(n+1) \Delta t$.

Initial conditions. The initial values for $\overline{\mathbf{v}}_{0}, \mathbf{v}_{0}$, and $\mathbf{v}_{1}$ are computed in the following way.

First, the one-mode solutions to the linearized form of the equations (5.2),

$$
\frac{\partial \overline{\mathbf{v}}_{0}}{\partial t}=\frac{1}{R} \Delta \overline{\mathbf{v}}_{0}, \quad \nabla \cdot \overline{\mathbf{v}}_{0}=0
$$

which are just $\bar{u}_{0}(t=0, y)=a_{1} \cos \left[\pi\left(k_{1}-1 / 2\right) y\right]$ and $\bar{w}_{0}(t=0, y)=a_{2} \cos \left[\pi\left(k_{2}-1 / 2\right) y\right]$, are taken as the initial condition for $\overline{\mathbf{v}}_{0}$. Then they are inserted into the quasi-linear equations (5.3) for $u_{0}, v_{0}$, and $w_{0}$, with the forcing terms containing $\mathbf{v}_{+}$and $\mathbf{v}_{-}$neglected. Since $u_{0}$ does not appear in the continuity equation in (5.3), its initial value is independent of those of $v_{0}$ and $w_{0}$. For this reason, the equation for $u_{0}$ and the equations for $v_{0}$ and $w_{0}$ in (5.3) are computed separately, and the term involving $v_{0}$ in the equation for $u_{0}$ is ignored. The initial conditions for $\mathbf{v}_{0}$ are computed in such a way that they are the least stable or most unstable eigenfunctions of the quasilinear eigenvalue problems derived from (5.3) by neglecting all the forcing terms in the equations. Then (5.4) is changed to an eigenvalue problem by using the initial values of $\overline{\mathbf{v}}_{0}$ and $\mathbf{v}_{0}$ (so that they are functions of $y$ only after the exponential terms are canceled), and the most least stable or most unstable eigenfunctions are used as the initial values for $\mathbf{v}_{+}$and $\mathbf{v}_{-}$.

Some simplifications may be made if we assume that $\bar{u}_{0}$ and $\bar{w}_{0}$ are even functions in $y$, and initially they have the forms

$$
\bar{u}_{0}=a_{1} \cos \left[\left(\pi k_{1}-\pi / 2\right) y\right], \quad \bar{w}_{0}=a_{2} \cos \left[\left(\pi k_{2}-\pi / 2\right) y\right] .
$$

Based on the even assumption for $\bar{u}_{0}$ and $\bar{w}_{0}, u_{0}, w_{0}, p_{0}, v_{+}$and $v_{-}$are assumed to be even in $y$ and $v_{0}, u_{+}, u_{-}, w_{+}, w_{-}, p_{+}$and $p_{-}$are assumed to be odd functions in $y$. It is easy to see from (5.2)-(5.4) that these assumptions are consistent. These simplifications are believed to be important in helping to assure the numerical accuracy of the computation.

Verification computations. A verification was made to investigate the truncation errors from the finite time difference in (A.2)-(A.4). First, the eigenvalue problem described above was solved by the Chebyshev tau and the Chebyshev collocation method. Then, taking the initial values from the least stable eigenmode from this eigenvalue computation and assuming that $\overline{\mathbf{v}}_{0}$ and $\mathbf{v}_{0}$ are functions of $y$ only, we computed (A.4) and compared the logarithm of the energy growth rate from this computation with that predicted by the eigenvalue computation.

The conditions of the comparison and the results are shown in Table A1, where $E n(t)=E n\left(\mathbf{v}_{+}, \mathbf{v}_{-}, t\right)$. It is seen that the time discrete error decreases as the time 


\begin{tabular}{|lllllll|}
\hline$R=1200$ & $\alpha=1$ & $\beta=2$ & $a_{1}=0.015$ & $k_{1}=5$ & & \\
$a_{2}=0.015$ & $k_{2}=3$ & $a_{3}=0.015$ & $a_{4}=0.01$ & $a_{5}=0.015$ & & \\
\hline$M=16$ & $t=$ & 2 & 4 & 6 & 8 & 10 \\
& $2 \lambda_{r} t$ & 0.1192 & 0.2384 & 0.3576 & 0.4768 & 0.5960 \\
\hline$\Delta t=0.4$ & $\log [\operatorname{En}(t)]$ & 0.1179 & 0.2358 & 0.3537 & 0.4717 & 0.5896 \\
$\Delta t=0.2$ & $\log [\operatorname{En}(t)]$ & 0.1188 & 0.2377 & 0.3567 & 0.4756 & 0.5944 \\
$\Delta t=0.1$ & $\log [\operatorname{En}(t)]$ & 0.1191 & 0.2382 & 0.3574 & 0.4765 & 0.5956 \\
$\Delta t=0.05$ & $\log [\operatorname{En}(t)]$ & 0.1192 & 0.2384 & 0.3575 & 0.4767 & 0.5959 \\
$\Delta t=0.01$ & $\log [\operatorname{En}(t)]$ & 0.1192 & 0.2384 & 0.3576 & 0.4768 & 0.5960 \\
\hline
\end{tabular}

TABLE A1

step $\Delta t$ becomes smaller, and for $\Delta t$ smaller than 0.05 , very good agreement is obtained. Since our purpose is to demonstrate the impact on the stability behavior when the basic flow is slightly modified, we feel that a computational result with an accuracy to the first three or four digits should be adequate. Therefore, $\Delta t=0.05$ is used for all the following computations. Also, both the Chebyshev tau and the Chebyshev collocation methods are used to ensure the correctness of the computational methods and the corresponding programs, and satisfactory results are observed.

\section{REFERENCES}

[1] M. Nishioka, S. Iida, and Y. Ichikawa, An experimental investigation of the stability of plane Poiseuille flow, J. Fluid Mech. 72, 731-751 (1975)

[2] L. D. Landau, On the vibrations of the electronic plasma, J. Phys. U.S.S.R. 10, 23-34 (1946)

[3] D. Meksyn and J. T. Stuart, Stability of viscous motion between parallel planes for finite disturbances, Proceedings of the Royal Society of London, Series A 208, 517-526 (1951)

[4] J. T. Stuart, On the non-linear mechanics of hydrodynamic stability, J. Fluid Mech. 4, 1-21 (1958)

[5] J. T. Stuart, On the non-linear mechanics of wave disturbances in stable and unstable parallel flows, Part 1, The basic behavior in plane Poiseuille flow, J. Fluid Mech. 9, 353-370 (1960)

[6] J. Watson, On the non-linear mechanics of wave disturbances in stable and unstable parallel flows, Part 2, The development of a solution for plane Poiseuille flow and for plane Couette flow, J. Fluid Mech. 9, 371-389 (1960)

[7] W. C. Reynolds and M. C. Potter, Finite-amplitude instability of parallel shear flows, J. Fluid Mech. 27, 465-492 (1967)

[8] C. L. Pekeris and B. Shkoller, Stability of plane Poiseuille flow to periodic disturbances of finite amplitude in the vicinity of the neutral curve, J. Fluid Mech. 29. 31-38 (1967)

[9] D. Grohne, Die Stabilität der ebenen Kanalströmung gegenuber dreidimensionalen Störungen von endlicher Amplitude, AVA Göttingen Report, 69-A-30 (1969)

[10] T. A. Zang and S. E. Krist, Numerical experiments on stability and transition in plane channel flow, Theoret. Comput. Fluid Dynamics 1, 41-64 (1989)

[11] T. Herbert, Periodic Secondary Motions in a Plane Channel, in Proc. Internat. Conf. Numer. Methods Fluid Dynamics, eds., A. I. van de Vooren and P. J. Zandbergen, Springer-Verlag, Berlin, 1976

[12] T. Herbert, Die neutrale Fläche der ebenen Poiseuille-Strömung, Habiltationsschrift. Univ. Stuttgart, W. Germany, 1978

[13] L. Wolf, Z. Lavan, and H. J. Nielsen, Numerical computation of the stability of plane Poiseuille flow, J. Appl. Mech. 45, 13-18 (1978)

[14] S. A. Orszag and A. T. Patera, Subcritical transition to turbulence in plane channel flows, Phys. Rev. Lett. 45, 989-993 (1980) 
[15] L. Kleiser and U. Schumann, Spectral simulations of the laminar-turbulent transition process in plane Poiseuille flow, in Spectral Methods for Partial Differential Equations, eds. R. G. Voight, D. Gottlieb, and M. Y. Hussaini, SIAM, Philadelphia, PA, 1984, pp. 141-164

[16] S. A. Orszag and L. C. Kells, Transition to turbulence in plane Poiseuille flow and plane Couette flow, J. Fluid Mech. 96, 159-205 (1980)

[17] S. A. Orszag and A. T. Patera, Secondary instability of wall-bounded shear flows, J. Fluid Mech. 128, 347-385 (1983)

[18] T. Herbert, On perturbation methods in nonlinear stability theory, J. Fluid Mech. 126, 167-186 (1983)

[19] B. J. Bayly, S. A. Orszag, and T. Herbert, Instability mechanisms in shear-flow transition, Annual Reviews of Fluid Mech. 20, 359-391 (1988)

[20] D. J. Benney and C. C. Lin, On the secondary motion induced by oscillations in a shear flow, Phys. Fluids 3, 656-657 (1960)

[21] P. S. Klebanoff, K. D. Tidstrom, and L. M. Sargent, The three-dimensional nature of a boundarylayer instability, J. Fluid Mech. 12, 1-34 (1962)

[22] D. J. Benney, The evolution of disturbances in shear flows at high Reynolds numbers, Stud. Appl. Math. 70, 1-19 (1984)

[23] R. C. DiPrima and J. T. Stuart, Hydrodynamic stability, J. Appl. Mech. 50, 983-991 (1983)

[24] M. Nishioka and M. Asai, Some observations of the subcritical transition in plane Poiseuille flow, J. Fluid Mech. 150, 441-450 (1985)

[25] O. Reynolds, An experimental investigation of the circumstances which determine whether the motion of water shall be direct or sinuous, and of the law of resistance in parallel channels, Philos. Trans. Roy. Soc. London 174, 935 (1883) 